\title{
Testing spore amyloidity in Agaricales under light microscope: the case study of Tricholoma
}

\author{
Alfredo Vizzini ${ }^{1 *}$, Giovanni Consiglio ${ }^{2}$ and Ledo Setti ${ }^{3}$
}

\begin{abstract}
Although species of the genus Tricholoma are currently considered to produce inamyloid spores, a novel standardized method to test sporal amyloidity (which involves heating the sample in Melzer's reagent) showed evidence that in the tested species of this genus, which belong in all 10 sections currently recognized from Europe, the spores are amyloid. In two species, T. josserandii and T. terreum, the spores are also partly dextrinoid. This result provides strong indication that a positive reaction of the spores in Melzer's reagent could be a character shared by all genera in Tricholomataceae s. str.
\end{abstract}

Keywords: Agaricomycetes, Basidiomycota, lodine, Melzer's reagent, nrlTS sequences, Pre-heating, Taxonomy of Tricholomataceae

\section{Introduction}

It has been known for about 150 years that some ascomycete and basidiomycete sporomata may contain elements which stain grey to blue-black with iodinecontaining solutions. Such a staining was termed amyloid reaction, sometimes written as $\mathrm{I}+$ or $\mathrm{J}+$ (the term "amyloid" being derived from the Latin amyloideus, i.e. starch-like), because plant starch gives a similar reaction with iodine (starch-reaction) (Bailey and Whelan 1961; Locquin and Langeron 1978; Immel and Lichtenthaler 2000). The blue colour of the stain is due to the amylose component of plant starch (Takahashi and Ono 1972; Bluhm and Zugenmaier 1981; Moulik and Gupta 1984; Murdoch 1992; Immel and Lichtenthaler 2000). The amylose chain forms a helix shape, and iodine (as triiodide anion $\mathrm{I}_{3}{ }^{-}$) can be bound inside the helix channel. The other component, amylopectin, gives a red-purple colour which is much less intense than the amylose stain (Bailey and Whelan 1961; Shen et al. 2013). The nature

\footnotetext{
* Correspondence: alfredo.vizzini@unito.it

'Department of Life Sciences and Systems Biology, University of Torino and Institute for Sustainable Plant Protection (IPSP-SS Turin), C.N.R, Viale P.A.

Mattioli, 25, I-10125 Torino, Italy

Full list of author information is available at the end of the article
}

of the starch-iodine interaction is extremely complex and still remains imperfectly known (Bluhm and Zugenmaier 1981; Immel and Lichtenthaler 2000; Shen et al. 2013; Du et al. 2014; Okuda et al. 2020).

An overview of the historical use of Melzer's was provided by Leonard (2006). Iodine was used in Mycology in the mid-1800s (as alcoholic solutions) mainly for studying lichens and asci (entire ascus wall or apical apparatus). The earliest reference to the use of such a bluing reaction in fungi as a character having a systematic significance is a report of the bluing of a cleistothecial marine ascomycete, Amylocarpus encephaloides by Currey (1859). Then the Tulasne brothers (1865), Nylander (1865) and Rolland (1887) noted and described iodine bluing in lichens and Ascomycetes at ascus and ascospore level. Boudier $(1885,1905-1910)$ was the first to describe and illustrate the use of iodine to classify apothecial Ascomycetes. The first reports of iodine solutions used in Basidiomycetes are those of Patouillard (1887), who described a violet coloration with iodine of the spores of Cyphella vitellina (now Aleurodiscus vitelli$n u s)$, and of Rolland (1887), who signaled a blue coloration in the stipe trama of Mycena tenerrima. At the

(c) The Author(s). 2020 Open Access This article is licensed under a Creative Commons Attribution 4.0 International License, which permits use, sharing, adaptation, distribution and reproduction in any medium or format, as long as you give appropriate credit to the original author(s) and the source, provide a link to the Creative Commons licence, and indicate if changes were made. The images or other third party material in this article are included in the article's Creative Commons licence, unless indicated otherwise in a credit line to the material. If material is not included in the article's Creative Commons licence and your intended use is not permitted by statutory regulation or exceeds the permitted use, you will need to obtain permission directly from the copyright holder. To view a copy of this licence, visit http://creativecommons.org/licenses/by/4.0/. 
time, however, such colour changes observed in microanatomical elements of Basidiomycetes were not considered to be systematically relevant by the above mentioned authors. As highlighted by Leonard (2006), it is worth noting that the following year Patouillard (1888) published a paper in which he stressed the importance of spore colour in the classification of fungi, even if he did not mention the use of iodine.

The turning point came when Melzer (1924), after studying Russula species for many years, developed a method based on the use of an iodine reagent mixed with chloral hydrate to clear and stain microscopical preparations, which made it possible to study the fine details of the sporal ornamentation of Russula species. Baral (1987a) believed that Melzer was "probably inspired by Arthur Meyer", a German botanist, renowned for his studies on chloroplasts, who introduced the use of chloral hydrate/iodine in Botany under the name "Chloraljod" (Meyer 1883). The formula for what became later known as Melzer's reagent - and which quickly came into standard use in mycology - is as follows: KI - $1.5 \mathrm{~g}$, iodine $-0.5 \mathrm{~g}$, distilled water $-20 \mathrm{~g}$, chloral hydrate $-22 \mathrm{~g}$ ). The resulting brownish solution stained dark black-blue the Russula sporal ornamentations.

With this reagent, Melzer supplied mycologists with an extraordinarily valuable tool, which has now become so widely used that the term Melzer's solution (or reagent or simply "Melzer's") appears in almost all taxonomic works on the higher fungi. At first, with the exception of some papers by Boursier (1925) and Kühner (1926a, b), Melzer's reagent was only used by Russula specialists because it was invaluable in allowing for an indepth study of the ornamentation of Russula spores (Crawshay 1930; Moreau 1930; Malençon 1931; Singer 1932).

However, this new reagent was soon also used for identification purposes in other groups of pale-spored agarics. Gilbert and Kühner (1928) highlighted that the spore wall is amyloid (blue) in some species of Amanita, but unreactive (or inamyloid, i.e. remaining pale yellow-brown or clear) in others. Positive iodine reactions of the spore wall were observed by Kühner (1931, 1936a, 1938) in many species of the genus Mycena and, by Kühner and Maire (1934), in many other white-spored agarics. Further, Metrod (1932), Kühner and Maire (1934) and Kühner (1936b) discovered that Melzer's reagent caused a different, purple-red staining in the spore wall of many species of the genus Lepiota and in the basidiome hyphae (especially those of the stipe in Mycena) of other agarics. Kühner and Maire (1934) and Kühner (1936a, b) used indiscriminately the term "amyloid" for both the blue and the purple-red reaction on elements of the basidiome. The dark red-brown reaction in Melzer's reagent was later named pseudoamyloidity by Singer $(1938,1951)$ and some decades later, dextrinoidity by Orton (1960).
Gilbert (1940) used the following modified solution for Amanita spores: distilled water $10 \mathrm{ml}, \mathrm{KI} 0.5 \mathrm{~g}$, iodine $0.05 \mathrm{~g}$, chloral hydrate $10 \mathrm{~g}$. This solution is almost colourless under the microscope, but the Amanita spore walls turn dark grey.

Langeron (1945), in the formulation of Melzers' s reagent included in his book, used $22 \mathrm{~g}$ of chloral hydrate instead of Melzer's $20 \mathrm{~g}$, but there was no discussion as to why he changed the proportions. This change in the proportions of Melzer's is now known as "Langeron's modification" (Ainsworth 1961) and was adopted by Singer (1951, 1963, 1975, 1986).

The contributions of further authors to the generalized use of the Melzer's reagent led to rapid progress in fungal taxonomy. This was not only especially significant in the taxonomy of the pale-spored agarics (Kühner and Romagnesi 1953; Moser 1967, 1978; Kühner 1980, 1984; Singer 1982, 1986; Horak 2005; Bas et al. 1988; Ludwig 2012; Knudsen and Vesterholt 2018), but it was also beneficial to other non-agaricoid fungal groups in Agaricomycotina (e.g., Imler 1950; Donk 1964; Miller 1964; Smith and Zeller 1966; Dodd 1972; Jülich and Stalpers 1980; Watling 1980; Jülich 1984; Hjortstam et al. 1988; Stalpers 1996; Ginns 1998; Ryvarden and Melo 2014; Agerer 2018) and Pezizomycotina (Kohn and Korf 1975; Nannfeldt 1976; Rossman 1980; Baral 1987a, b, 2007, 2009; Common 1991; Frey 2016).

In some papers (Kohn and Korf 1975; Nannfeldt 1976; Redhead 1977; Baral 1987a, b, 2007, 2009) the importance of using a $\mathrm{KOH}$ pretreatment of the sample to enforce amyloid reactions in ascal tips was stressed. Baral (1987a, b, 2007, 2009) highlighted that Lugol's, a chloral hydrate-free iodine solution developed in 1829 by the French physician J.G.A. Lugol (initially as a cure for tuberculosis, Calissendorff and Falhammar 2017) is more efficient than Melzer's in inducing amyloid reactions on apical apparatus in asci.

Iodine solutions (Lugol's and Melzer's) are known in Agaricomycetes to induce amyloid reactions on other structures besides the spores (Agerer 1999) such as, for example, the hypodermium and pileus and lamellar trama of some Mycena species (Kühner 1938), the hymenial cystidia of Amylocystis (Singer 1944) and many Tubulicrinis (Donk 1964), context hyphae of some Cortinarius species (sect. Purpurascentes, Moser 1961), hyphae of the stipe base in some Boletus species (Imler 1950; Singer 1965; Vizzini et al. 2014), elements in the ectomycorrhizae of Chroogomphus and Rhizopogon (Smith and Zeller 1966; Agerer 1990; Scambler et al. 2018).

The chemical components of Melzer's reagent have different functions. Chloral hydrate, being a clearing agent, clarifies and improves the transparency of various dark-colored microscopic materials. Iodine is hardly soluble in water, therefore, potassium iodide is used to 
improve its solubility (thanks to the reaction $\mathrm{I}_{2}+\mathrm{I}^{-}=$ $\mathrm{I}_{3}{ }^{-}$); iodine is thought to be the main active staining agent in Melzer's, even if its mechanism of action in the amyloid reaction is not entirely understood. It is thought to react/interact with starch-like polysaccharides present in the walls of spores and hyphae, but data in this regard are dated and incomplete (Smith 1965; Blackwell et al. 1985; Morton 1986; Leonard 2006). McCracken and Dodd 1971, Dodd and McCracken (1972) and McCracken et al. (1973) noted that fungal "starch" is different from plant starch in that it is not produced in plastids, is not in granular form, is mainly a cell-wall component (rather than an energy source), and is made up of "only short-chained amylose molecules." They hypothesized that the amylose in the spore cell helped the spore stay viable until conditions were good for germination. Webster and Weber (2007) suggested that the amylose-like substances prevent oxygen from entering the spore, thereby enforcing dormancy by slowing down metabolism, a hypothesis re-proposed by Halbwachs and Bässler (2015) in their review of spore morphology in agarics. Amylose is insoluble in water and also more resistant to digestion compared to other forms of starch (i.e. amylopectin) (Chen 1990; Bertoft 2017), which could then also contribute to a longer spore dormancy.

A completely different cell wall composition should be the basis of dextrinoidity. Blackwell et al. (2001) suggested that the red-brown reaction with Melzer's reagent does not involve starch or amylose, but is a reaction with glycine betaine, an osmolyte (an organic osmotic solute) which they found in high concentrations in the Basidiomycetes they studied (Antrodia carbonica, Calvatia sp., Chlorophyllum molybdites, Ganoderma lucidum and Laetiporus sulphureus).

Focusing on the spores of the pale-spored agarics, the amyloid positive reactions obtained with this medium range from pale grey to blue to violet-black (Meixner 1975; Locquin and Langeron 1978; Erb and Matheis 1983; Brunori et al. 1985; Singer 1986; Charbonnel 2004; Basso 2005). The solution can be stored for a long time (Ammirati et al. 1985; Clémençon 2000, 2012; Lecomte 2017). Usually a positive reaction occurs almost immediately; when the reaction is doubtful, leaving the material in solution for $30 \mathrm{~min}$ is recommended (Clémençon 2012). The amyloid reaction may not be of the same intensity on the entire surface of a spore (e.g. Mycena, Gloiothele, H. Clémençon, pers. comm.). Fresh material sometimes gives a slower reaction than dried material. Melzer's cannot be used in combination with alkali because iodine reacts with hydroxide ions and a cloudy precipitate develops. Consequently, when potassium hydroxide or ammonium are used as a pretreatment, the alkalinity must be first neutralized before adding Melzer's (Largent et al. 1977; Ammirati et al. 1985).
The amyloidity of spore-wall was used as a distinguishing taxonomical character at family (i.e. Bondarzewiaceae, Russulaceae), subfamily/tribus (i.e. Leucopaxilloideae/Leucopaxilleae), genus (i.e. Leucopaxillus, Xeromphalina, Pseudomphalina), subgenus (i.e. Amanita, Fayodia), section level (i.e. Cystoderma, Dermoloma, Hydropus, Mycena). Quite recently, the genus Cystodermella has been segregated from Cystoderma for the species with inamyloid spores (Harmaja 2002), a proposition later supported by molecular analysis (Saar et al. 2009). A curious exception concerning amyloid reactions is Mycena sect. Calodontes, which encompasses both amyloid and inamyloid spore-producing species. In fact, one of its species, $M$. pearsoniana, can show either amyloid or inamyloid reactions interchangeably (Harder et al. 2012). Therefore, presence/absence of an amyloid reaction is clearly a homoplastic character and cannot be used in species delimitation in this section.

At present, it is also known that the amyloid reaction of spore ornamentations is a character that may have evolved several times. Compelling evidence for this is offered by Leucopaxillus and Melanoleuca, genera traditionally included in the tribe Leucopaxilleae or subfamily Leucopaxilloideae of the Tricholomataceae (Singer 1986; Bon 1991) but which recent molecular studies (Matheny et al. 2006; Dentinger et al. 2016) now place in the tricholomatoid clade (suborder Tricholomatineae) and in the Pluteoid clade (suborder Pluteineae), respectively.

The amyloid reaction of the spores can sometimes be so weak as to be difficult to assess properly [e.g. in Pseudoclitocybe (Bigelow 1982); some species of Mycena sect. Calodontes (Harder et al. 2012); Pseudolaccaria (Lavorato et al. 2015); Musumecia (Li et al. 2016] and, consequently, there might be some subjectivity on the part of the operator. To avoid the possible distorting consequences of subjectivity in the assessment of this character, a novel standardized method to test sporal amyloidy has recently been used by the present authors (Alvarado et al. 2018). It allowed us to obtain unambiguous results in cases (e.g. in Pseudoclitocybaceae, Alvarado et al. 2018) in which the traditional testing method led to results of dubious interpretation. Based on these outcomes, a project was set up to test with this new method amyloid reactions in the spores of Agaricales when observed under light microscopy. It was decided to start, in the present paper, with the species of the large genus Tricholoma, typified with Tricholoma flavovirens (= T. equestre), a widely distributed genus of ectomycorrhizal agarics that was traditionally considered to produce inamyloid spores (Kühner and Maire 1934; Bon 1984, 1991; Singer 1986; Riva 1988, 2003; Christensen and Heilmann-Clausen 2013). However, it has recently been shown to belong to a clade (Tricholomataceae s. stricto) containing mostly taxa with positive reaction of the spores in Melzer’s reagent (Sánchez-García et al. 2014). 


\section{Materials and methods Morphology}

The eighteen Tricholoma collections (corresponding to seventeen species) used for the microscopic analyses (Fig. 1, Table 1) were identified using the monographic work by Christensen and Heilmann-Clausen (2013) and were selected to represent all the ten sections of Tricholoma recognized in Christensen and Heilmann-Clausen (2013) and Heilmann-Clausen et al. (2017): Atrosquamosa, Caligata, Contextocutis (= Saponacea), Genuina, Lasciva, Megatricholoma, Pardinicutis, Sericella (= Sericeocutis), Terrea and Tricholoma. Collections of Tricholoma apium, $T$. arvernense, $T$. fucatum and $T$. josserandii, species which occupy an isolate position in Heilmann-Clausen et al. (2017), were also studied. Seventeen of these collections have been deposited at $\mathrm{AMB}$ and sequenced in the present work, while for one, T. lascivum C-F-96230 (shown in Fig. 1 with an asterisk), the nrITS sequence was already present in the public databases GenBank (https://www.ncbi.nlm.nih.gov/ genbank/) and UNITE (https://unite.ut.ee/) (LT000028, UDB000005; Heilmann-Clausen et al. 2017). A standardized protocol to test spore amyloidity (named here as the heating method, HM) was applied (used for the first time by the present Authors in Alvarado et al. 2018 and Vizzini et al. 2020): a lamellae sample was hydrated in tap water for $1-2 \mathrm{~h}$ or $5 \%$ ammonia, and then heated in a drop of Melzer's reagent (original formulation, Melzer 1924) in a stainless steel spoon until reaching the boiling point at least 1 or 2 times. The sample immersed in Melzer's was immediately removed from the heat and transferred to a flat Teflon surface, cut into three or four pieces, and transferred again to a slide with a drop of fresh Melzer's reagent. The excess liquid was then removed and the sample gently squashed and examined with a $60 \times$ or $100 \times$ bright field objective (numerical aperture NA 1.3, diaphragm open to about $50 \%$ range, aperture field 0.9). The classical method (CM) without pre-heating was also used under a different set of conditions: a lamellae sample was hydrated in tap water for $1-2 \mathrm{~h}$ or soaked for $3 \mathrm{~min}$. in $5 \%$ ammonia, or in $5 \%$ $\mathrm{KOH}$, washed and then transferred into Melzer's, squashed and observed immediately after squashing (CMi), after $30 \mathrm{~min}$. (CM30) and $120 \mathrm{~min}$. (CM120). Furthermore, two species of the genus Amanita, A. phalloides (subg. Lepidella, AMB 18719) and A. argentea (subg. Amanita, AMB 18720), traditionally recognized as having clearly evidently amyloid and inamyloid spores, respectively (Neville and Poumarat 2004; Tulloss 2020; Tulloss and Possiel 2020), were tested with all the methods (under all the different conditions) above mentioned. These species were chosen as an additional control for the new proposed method. A spore print on glass (microscope slides) obtained from a basidiome of
T. josserandii (TO AV260920) was tested for amyloidity adding directly a drop of Melzer's and observed after complete absorption/evaporation of the reagent $(2 \mathrm{~h})$ (Fig. 8).

Microscopic pictures were taken on a Canon Power Shot A640 digital camera connected to a Zeiss Axioscope 40 microscope with both interferential contrast and phase-contrast optics.

\section{DNA extraction, amplification and sequencing}

Total DNA was extracted from dry specimens (Table 2) employing a modified protocol based on Murray and Thompson (1980). PCR amplification (Mullis and Faloona 1987) included 35 cycles with an annealing temperature of $54^{\circ} \mathrm{C}$. Primers ITS1F and ITS4 (White et al. 1990; Gardes and Bruns 1993) were employed for the region. PCR products were checked in $1 \%$ agarose gels, and positive reactions were sequenced with one or both PCR primers. Chromatograms were checked searching for putative reading errors, and these were corrected. The accession numbers of the sequences are reported in Table 2.

\section{Phylogenetic analyses}

The nrITS dataset was assembled based on that of Heilmann-Clausen et al. (2017). BLASTn (Altschul et al. 1990) was used to select the most closely related sequences from GenBank and UNITE. Hypsizygus marmoreus (HM561970) was used as outgroup taxon to root the tree.

Sequences first were aligned in MEGA 6.0 software (Tamura et al. 2013) with its MUSCLE application (Edgar 2004) and then corrected manually. The nrITS alignment was not partitioned into ITS1, 5.8S and ITS2 regions. The Bayesian analysis was performed through the CIPRES Science Gateway platform (Miller et al. 2010) by using the MrBayes v. 3.2.7 algorithm with two simultaneous runs, four chains, temperature fixed at 0.2 and sampling every 1000 generations until reaching the convergence parameters (standard deviation less than 0.01). The first $25 \%$ trees were discarded as burn-in. Finally, a full search for the best-scoring Maximum likelihood tree was performed in RAxML v.8.2.10 (Stamatakis 2014) using the standard search algorithm (GTRCAT model, 2000 bootstrap replications). Significance threshold was set $\geq 0.95$ for posterior probability (PP) and $\geq 70 \%$ for bootstrap proportions (BP).

\section{Results}

The correct determination of all the Tricholoma collections used in this work was confirmed molecularly (Fig. 2). The ten sections (major clades) recognized in Heilmann-Clausen et al. (2017) are also recovered in the present analysis. Tricholoma apium, T. arvernense, $T$. fucatum and T. josserandii are not included in these sections and occupy an isolated position. 

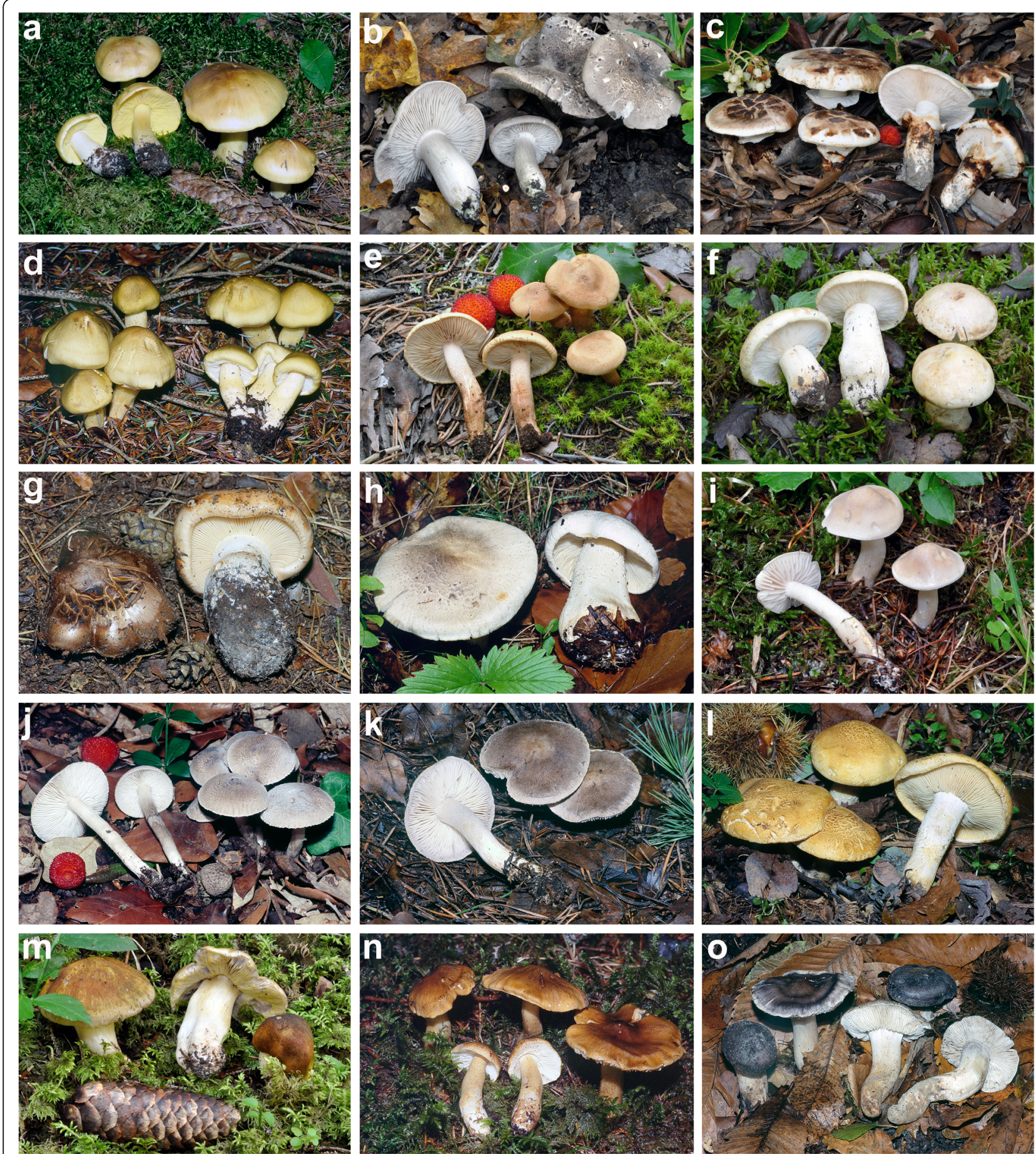

Fig. 1 Tricholoma collections examined. a T. frondosae (AMB 17243) b T. orirubens (AMB 17410) c T. caligatum (AMB 17231) d T. saponaceum (AMB 17433) e T. psammopus (AMB 17411) $\mathbf{f} T$. acerbum (AMB 17177) $\mathbf{g}$ T. colossus (AMB 17237) $\mathbf{h}$ T. filamentosum (AMB 17248) $\mathbf{i} T$. inamoenum (AMB 17367) $\mathbf{j}$ T. ramentaceum (AMB 17423) $\mathbf{k}$ T. terreum (AMB 17444) I T. apium (AMB 17203) $\mathbf{m}$ T. arvernense (AMB 17215) $\mathbf{n}$ T. fucatum (AMB 17300) $\mathbf{o}$ T. josserandii (AMB 17407). Photos by G. Consiglio

All the Tricholoma collections showed spores with at least partly dark grey to blue amyloid walls (apiculus included) under the light microscope both by the novel standardized protocol $(\mathrm{HM})$ and the classical method with spores observed after $30 \mathrm{~min}$. (CM30) and $120 \mathrm{~m}$. (CM120), regardless of the soaking medium used (Table 1) (Figs. 3 and $4 \mathrm{c}-\mathrm{i}$ ). The amyloid reaction was also observed on the apical part of basidia (Figs. 3a-d and 4g, i) with both HM and CM30 
Table 1 Spore reaction in Melzer's depending on the method used

\begin{tabular}{|c|c|c|c|c|}
\hline Species & $\mathrm{CMi} \mathrm{H}_{2} \mathrm{O} / \mathrm{NH}_{3} / \mathrm{KOH}$ & $\mathrm{CM} 30 \mathrm{H}_{2} \mathrm{O} / \mathrm{NH}_{3} / \mathrm{KOH}$ & $\mathrm{CM} 120 \mathrm{H}_{2} \mathrm{O} / \mathrm{NH}_{3} / \mathrm{KOH}$ & $\mathrm{HM} \mathrm{H}_{2} \mathrm{O} / \mathrm{NH}_{3}$ \\
\hline Amanita argentea & inamyloid & inamyloid & inamyloid & inamyloid \\
\hline Amanita phalloides & amyloid & amyloid & amyloid & amyloid \\
\hline Tricholoma acerbum & inamyloid & amyloid & amyloid & amyloid \\
\hline Tricholoma apium & inamyloid & amyloid & amyloid & amyloid \\
\hline Tricholoma argyraceum & inamyloid & amyloid & amyloid & amyloid \\
\hline Tricholoma arvernense & inamyloid & amyloid & amyloid & amyloid \\
\hline Tricholoma caligatum & inamyloid & amyloid & amyloid & amyloid \\
\hline Tricholoma colossus & inamyloid & amyloid & amyloid & amyloid \\
\hline Tricholoma filamentosum & inamyloid & amyloid & amyloid & amyloid \\
\hline Tricholoma frondosae & inamyloid & amyloid & amyloid & amyloid \\
\hline Tricholoma fucatum & inamyloid & amyloid & amyloid & amyloid \\
\hline Tricholoma inamoenum & inamyloid & amyloid & amyloid & amyloid \\
\hline Tricholoma josserandii & inamyloid & dextrinoid/amyloid & dextrinoid/amyloid & dextrinoid/amyloid \\
\hline Tricholoma lascivum & inamyloid & amyloid & amyloid & amyloid \\
\hline Tricholoma orirubens & inamyloid & amyloid & amyloid & amyloid \\
\hline Tricholoma psammopus & inamyloid & amyloid & amyloid & amyloid \\
\hline Tricholoma ramentaceum & inamyloid & amyloid & amyloid & amyloid \\
\hline Tricholoma saponaceum & inamyloid & amyloid & amyloid & amyloid \\
\hline Tricholoma terreum & inamyloid & dextrinoid/amyloid & dextrinoid/amyloid & dextrinoid/amyloid \\
\hline
\end{tabular}

CMi spores observed in Melzer's immediately after squashing and without preheating, CM30 spores observed after leaving the sample in Melzer's for 30 min and without preheating, $C M 120$ spores observed after leaving the sample in Melzer's for 120 min and without preheating, $H M$ spores observed in Melzer's after heating, $\mathrm{H}_{2} \mathrm{O} / \mathrm{NH}_{3} / \mathrm{KOH}$ soaking media

methods. The classical method with spores observed in Melzer's immediately after squashing $(\mathrm{CMi})$ always produced negative or difficult to interpret results regardless of the soaking medium used (Table 1) (Figs. 4a-b, 5a-b and 6a-b). Spores of Tricholoma josserandii and T. terreum, which were negative with the CMi method (Table 1) (Figs. 5a-b and $6 a-b)$ showed an evident dextrinoid reaction (Figs. 5c-f and $6 \mathrm{c}-\mathrm{f}$ ) coupled or not with an amyloid plaque reaction (Fig. $3 \mathrm{c}-\mathrm{d}, \mathrm{j}$ ) only with the $\mathrm{HM}$ and CM30 methods, regardless of the soaking medium used. The spore print of $T$. josserandii is amyloid (Fig. 8c).

Spores of Amanita phalloides are clearly amyloid (Fig. 7ae) and spores of $A$. argentea are inamyloid (Fig. $7 \mathrm{f}-\mathrm{h}$ ) by whatever method is used. Some apically amyloid basidia are also present in A. phalloides (Fig. 7b-d).

\section{Specimens examined}

TRICHOLOMA. Sect. Atrosquamosa - Tricholoma orirubens. Italy: Emilia-Romagna, Bologna, Lizzano in Belvedere, Vidiciatico, Rio Ri, in a mixed wood of broadleaved trees, 04 Oct. 1993, G. Consiglio (AMB 17410). Sect. Caligata - Tricholoma caligatum. Italy: Lazio, Roma, Santa Marinella, Piano delle Tavole, under Quercus ilex, Pinus halepensis and Arbutus unedo, 13 Nov. 2015, G. Consiglio \& G. Simonini (AMB 17231). Sect. Contextocutis - Tricholoma saponaceum. Italy: Emilia-
Romagna, Bologna, Lizzano in Belvedere, Vidiciatico, Rio Ri, under Fagus sylvatica and Abies alba, 13 Oct. 2001, G. Consiglio \& G. Spisni (AMB 17433). Sect. Genuina - Tricholoma psammopus. Italy: Toscana, Siena, Castellina in Chianti, Cipresseta di S. Agnese, under Cupressus sempervirens and Arbutus unedo, 19 Nov. 2015, G. Consiglio \& E. Franceschini (AMB 17411). Sect. Lasciva - Tricholoma lascivum. Denmark: Northern Zealand, Rude Skov, Maglebjerg, under Fagus sylvatica, 17 Sept. 2000, M. Christensen (C-F 96230, MC00-519). Sect. Megatricholoma - Tricholoma acerbum. Italy: Emilia-Romagna, Bologna, Monterenzio, Ronchi, in a mixed wood of broadleaved trees, 28 Oct. 1992, G. Consiglio (AMB 17177). Tricholoma colossus. Italy: Trentino, Trento, Baselga di Piné, Laghestèl, under Pinus sylvestris, 03 Oct. 2002, G. Consiglio, G. Marasca \& B. Oss-Emer (AMB 17237). Sect. Pardinicutis - Tricholoma filamentosum. Italy: Emilia-Romagna, Bologna, Lizzano in Belvedere, Vidiciatico, Rio Ri, under Fagus sylvatica and Abies alba, 04 Oct. 1993, G. Consiglio \& G. Spisni (AMB 17248). Sect. Sericella - Tricholoma inamoenum. Italy: Trentino, Trento, Baselga di Piné, Laghestèl, under Pinus sylvestris, 30 Aug. 2007, G. Consiglio, G. Marasca \& B. Oss-Emer (AMB 17367). Sect. Terrea - Tricholoma argyraceum. Italy: Marche, Pesaro-Urbino, Piobbico, Monte Grino, in a plantation of Cedrus atlantica, 
Table 2 Collections used for the phylogenetic analysis. Newly sequenced collections are in bold

\begin{tabular}{|c|c|c|c|c|c|}
\hline Species & Voucher & Herbarium no. & Country & GenBank accession no. & Unite accession no. \\
\hline Hypsizygus marmoreus & HM561970 & - & Malaysia & HM561970 & - \\
\hline Tricholoma acerbum & GC & AMB 17177 & Italy & MT462629 & - \\
\hline Tricholoma acerbum & JV99-638 & C-F-41483 & Denmark & LT000005 & UDB001474 \\
\hline Tricholoma acerbum & MC00-204 & C-F-96223 & Slovenia & LT000134 & UDB002361 \\
\hline Tricholoma acerbum & AF377247 & - & Norway & AF377247 & - \\
\hline Tricholoma apium & GC & AMB 17203 & Italy & MT462630 & - \\
\hline Tricholoma apium & JHC95-049 & C-F-35189 & Sweden & LT000154 & UDB001467 \\
\hline Tricholoma apium & JV00-215 & C-F-41884 & Denmark & LT000009 & UDB001685 \\
\hline Tricholoma apium & MC98-034 & C-F-59207 & Norway & LT000118 & - \\
\hline Tricholoma argyraceum & GC & AMB 17211 & Italy & MT462631 & - \\
\hline Tricholoma argyraceum & GC & AMB 17441 & Italy & MT462632 & - \\
\hline Tricholoma argyraceum & JHC95-112 & C-F-35092 & Denmark & LT000010 & UDB000780 \\
\hline Tricholoma argyraceum & JHC96-244 & C-F-96212 & Denmark & LT000011 & UDB000781 \\
\hline Tricholoma argyraceum & JHC97-092 & C-F-96213 & Sweden & LT000155 & UDB000782 \\
\hline Tricholoma argyraceum & MEN9491 & L0374886 epitype & The Netherlands & LT000198 & UDB000785 \\
\hline Tricholoma arvernense & GC & AMB 17215 & Italy & MT462633 & - \\
\hline Tricholoma arvernense & MC95-102 & C-F-59014 & Sweden & LT000157 & - \\
\hline Tricholoma arvernense & MC98-020 & C-F-59200 & Norway & LT000119 & UDB002362 \\
\hline Tricholoma arvernense & MC98-120 & C-F-59255 & France & LT000078 & UDB001438 \\
\hline Tricholoma caligatum & GC & AMB 17231 & Italy & MT462634 & - \\
\hline Tricholoma caligatum & KC565866 & - & Algeria & KC565866 & - \\
\hline Tricholoma caligatum & JV07-451 & C-F-76630 & Spain & LT000152 & - \\
\hline Tricholoma cingulatum & MC03-252 & C-F-96246 & Slovakia & LT000128 & UDB001420 \\
\hline Tricholoma cingulatum & MC96-134 & C-F-59057 neotype & Denmark & LT000015 & UDB000543 \\
\hline Tricholoma cingulatum & MC96-170 & C-F-59068 & Denmark & LT000016 & UDB000544 \\
\hline Tricholoma colossus & GC & AMB 17237 & Italy & MT462635 & - \\
\hline Tricholoma colossus & MC01-205 & C-F-96238 & Slovenia & LT000137 & UDB001417 \\
\hline Tricholoma colossus & MC97-047 & C-F-59154 & Sweden & LT000164 & UDB001433 \\
\hline Tricholoma equestre & MC94-027 & C-F-58886 & Denmark & LT000018 & UDB001508 \\
\hline Tricholoma equestre & MC95-187 & C-F-96256 & Denmark & LT000019 & - \\
\hline Tricholoma equestre & MC96-155 & C-F-58890 & Denmark & LT000020 & UDB001469 \\
\hline Tricholoma filamentosum & GC & AMB 17248 & Italy & MT462636 & - \\
\hline Tricholoma filamentosum & C-F35924 & C-F-35924 & Sweden & LT000165 & UDB001506 \\
\hline Tricholoma filamentosum & JHCO-1202 & C-F-96191 & Slovenia & LT000138 & UDB000804 \\
\hline Tricholoma filamentosum & MCO0-218 & C-F-96226 & Slovenia & LT000139 & - \\
\hline Tricholoma filamentosum & MCO3-242 & C-F-96243 & Slovakia & LT000129 & UDB000803 \\
\hline Tricholoma frondosae type I & AF349689 & - & USA & AF349689 & - \\
\hline Tricholoma frondosae type I & MC95-130 & C-F-59031 & Sweden & LT000167 & - \\
\hline Tricholoma frondosae type I & MC97-151 & C-F-59188 & Sweden & LT000168 & - \\
\hline Tricholoma frondosae type II & GC & AMB 17243 & Italy & MT462637 & - \\
\hline Tricholoma frondosae type II & MCO0-225 & C-F-96227 & Slovenia & LT000140 & - \\
\hline Tricholoma frondosae type II & MC96-235 & C-F-59084 & Denmark & LT000023 & UDB001509 \\
\hline Tricholoma frondosae type II & MC97-158 & C-F-59395 & Sweden & LT000169 & UDB002363 \\
\hline Tricholoma fucatum & GC & AMB 17300 & Italy & MT462638 & - \\
\hline
\end{tabular}


Table 2 Collections used for the phylogenetic analysis. Newly sequenced collections are in bold (Continued)

\begin{tabular}{|c|c|c|c|c|c|}
\hline Species & Voucher & Herbarium no. & Country & GenBank accession no. & Unite accession no. \\
\hline Tricholoma fucatum & MC97-149 & C-F-58980 neotype & Sweden & LT000170 & - \\
\hline Tricholoma fucatum & MC98-023 & C-F-59201 & Norway & LT000121 & - \\
\hline Tricholoma inamoenum & GC & AMB 17367 & Italy & MT462639 & - \\
\hline Tricholoma inamoenum & JHC95-042 & C-F-35182 neotype & Sweden & LT000173 & UDB001688 \\
\hline Tricholoma inamoenum & MC95-115 & C-F-59020 & Sweden & LT000174 & UDB001424 \\
\hline Tricholoma josserandii & GC & AMB 17407 & Italy & MT462640 & - \\
\hline Tricholoma josserandii & MC99-053 & C-F-96266 & France & LT000081 & UDB000797 \\
\hline Tricholoma josserandii & MC99-056 & C-F-96267 & France & LT000082 & UDB000798 \\
\hline Tricholoma lascivum & $\mathrm{JHCO3-020}$ & C-F-96194 & Slovakia & LT000131 & UDB001696 \\
\hline Tricholoma lascivum & MCO0-519 & C-F-96230 & Denmark & LT000028 & UDB000005 \\
\hline Tricholoma lascivum & MC99-197 & C-F-59446 & Denmark & LT000029 & - \\
\hline Tricholoma orirubens & GC & AMB 17410 & Italy & MT462641 & - \\
\hline Tricholoma orirubens & JHCO1-200 & C-F-96189 & Slovenia & LT000141 & UDB000524 \\
\hline Tricholoma orirubens & JHC93-261 & C-F-96208 & Denmark & LT000030 & UDB000523 \\
\hline Tricholoma orirubens & MC03-243 & C-F-96244 & Slovakia & LT000132 & UDB000801 \\
\hline Tricholoma orirubens & MC96-301 & C-F-59365 & Italy & LT000107 & UDB000522 \\
\hline Tricholoma pardinum & $J H C-01201$ & C-F-96190 & Slovenia & LT000142 & UDB000802 \\
\hline Tricholoma psammopus & GC & AMB 17411 & Italy & MT462642 & - \\
\hline Tricholoma psammopus & MC04-600 & C-F-96248 & Slovenia & LT000145 & - \\
\hline Tricholoma psammopus & MC96-345 & C-F-59324 & Italy & LT000108 & - \\
\hline Tricholoma psammopus & MC98-048 & C-F-59212 & Denmark & LT000036 & UDB001472 \\
\hline Tricholoma psammopus & MC99-089 & C-F-96273 & France & LT000084 & UDB001503 \\
\hline Tricholoma ramentaceum & GC & AMB 17423 & Italy & MT462643 & - \\
\hline Tricholoma ramentaceum var. pseudotriste & HQ184102 & - & France & HQ184102 & - \\
\hline Tricholoma saponaceum & GC & AMB 17433 & Italy & MT462644 & - \\
\hline Tricholoma saponaceum & C-F23337 & C-F-23337 & Denmark & LT000038 & UDB001499 \\
\hline Tricholoma saponaceum & JHCOO-049 & C-F-96188 & Norway & LT000123 & UDB001693 \\
\hline Tricholoma saponaceum & JHCO3-015 & C-F-96192 & Slovakia & LT000133 & UDB001694 \\
\hline Tricholoma saponaceum & JHCO4-429 & C-F-96196 & Sweden & LT000185 & UDB001697 \\
\hline Tricholoma scalpturatum & JHC93-263 & C-F-96210 & Denmark & LT000042 & UDB000541 \\
\hline Tricholoma scalpturatum & JHC94-231 & C-F-35309 & Denmark & LT000043 & UDB000542 \\
\hline Tricholoma scalpturatum & MC00-207 & C-F-96225 & Slovenia & LT000146 & - \\
\hline Tricholoma scalpturatum & MC95-165 & C-F-59399 neotype & Sweden & LT000187 & - \\
\hline Tricholoma terreum & GC & AMB 17444 & Italy & MT462645 & - \\
\hline Tricholoma terreum & JHC93-260 & C-F-96207 & Denmark & LT000057 & UDB000536 \\
\hline Tricholoma terreum & JHC95-118 & C-F-35098 & Denmark & LT000058 & - \\
\hline Tricholoma terreum & JHC95-172 & C-F-35154 & Denmark & LT000059 & UDB000812 \\
\hline Tricholoma terreum & MEN95192 & L0374887 neotype & Germany & LT000098 & UDB000813 \\
\hline
\end{tabular}

Cedrus deodara, Cupressus arizonica and Cupressus sempervirens, 04 Oct. 2007, G. Consiglio \& M. Maletti (AMB 17211); Lombardia, Mantova, Suzzara, under Quercus pubescens, 19 Nov. 2015, L. Setti (AMB 17441). Tricholoma orirubens. Italy: Emilia-Romagna, Bologna, Lizzano in Belvedere, Vidiciatico, Rio $\mathrm{Ri}$, in a mixed wood of broadleaved trees, 04 Oct. 1993, G. Consiglio (AMB 17410). Tricholoma ramentaceum. Italy: Toscana, Firenze, Empoli, La Striscia, under Quercus ilex and Arbutus unedo, 21 Nov. 2003, G. Consiglio \& E. Franceschini (AMB 17423). Tricholoma terreum. Italy: Emilia-Romagna, Bologna, Sasso Marconi, Prati di 


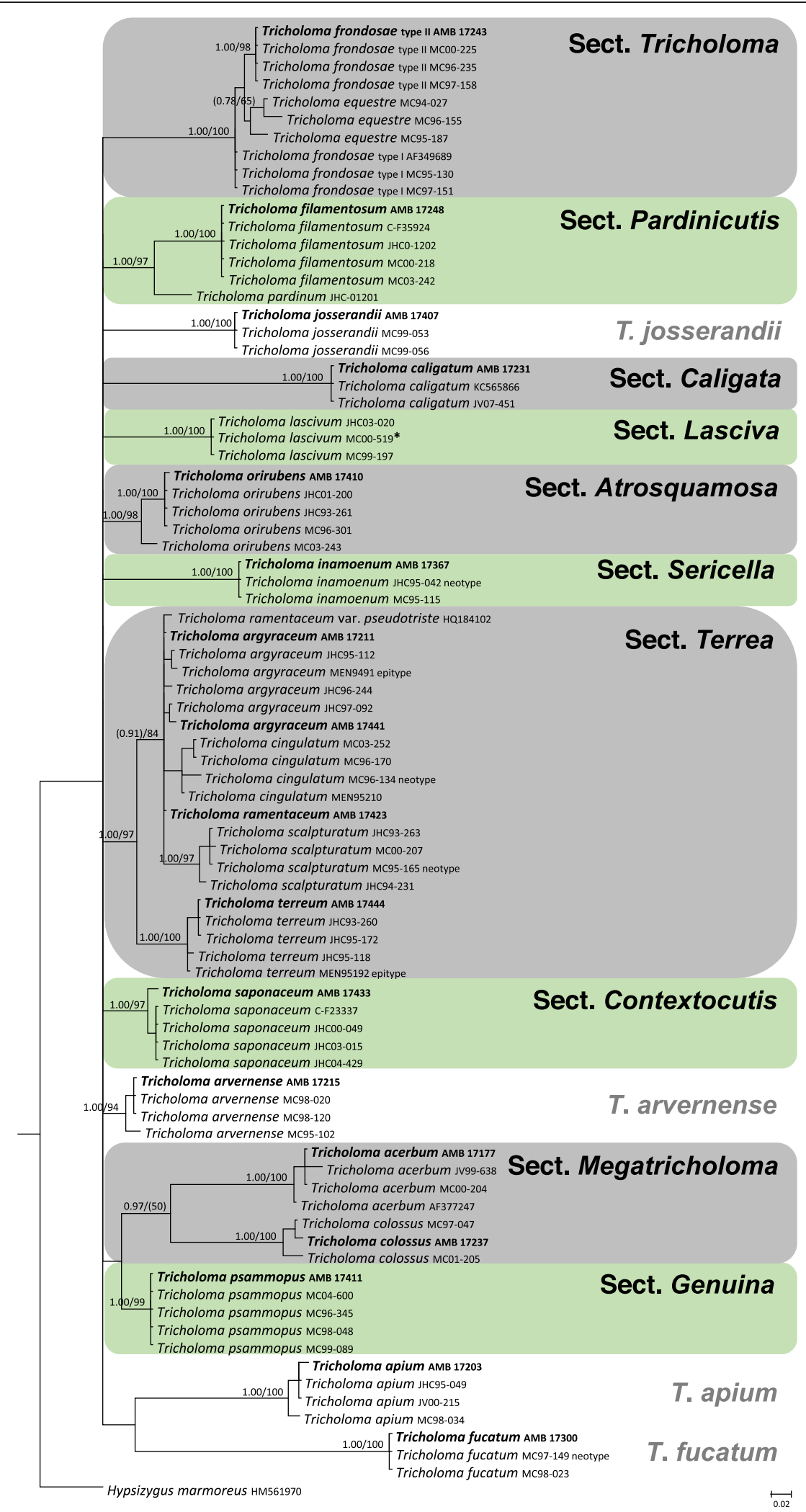

Fig. 2 (See legend on next page.) 
(See figure on previous page.)

Fig. 2 50\% majority rule ITS rDNA consensus phylogram of selected Tricholoma species (with Hypsizygus marmoreus as outgroup) obtained in MrBayes from 114,902 sampled trees. Nodes were annotated if supported by $\geq 0.95$ Bayesian PP (left) and/or $\geq 70 \%$ ML BP (right). Non-significant support values are exceptionally represented inside parentheses. Newly sequenced collections used in the morphological analysis are boldfaced. *indicates an additional collection used for testing spore amyloidity

Mugnano, under Pinus nigra, 10 Jan. 1998, G. Consiglio E G. Spisni (AMB 17444). Sect. Tricholoma - Tricholoma frondosae. Italy: Trentino, Trento, Predazzo, Parco di Paneveggio, under Picea abies, 24 Sept. 2010, G. Consiglio E G. Perdisa (AMB 17243). Incertae sedis - Tricholoma apium. Italy: Emilia-Romagna, Bologna, Gaggio Montano, Ronchidoso, in a mixed wood with Castanea sativa and Pinus sylvestris, 30 Sept. 1998, G. Consiglio (AMB 17203). Tricholoma arvernense. Italy: Trentino, Val di Pejo, Malga Torbi, under Abies alba, 30 Aug. 2013, G. Consiglio, M. Maletti, A. De Angelis E L. Polidori (AMB 17215). Tricholoma fucatum. Italy: Trentino, Trento, Predazzo, Bellamonte, under Picea abies, 19 Aug. 1998, G. Consiglio (AMB 17300). Tricholoma josserandii. Italy: Emilia-Romagna, Bologna, Grizzana Morandi, Veggio, Tudiano, under Castanea sativa, 27 Oct. 1999, G. Consiglio (AMB 17407); Piemonte, Torino, Pinerolo, Colle Pra Martino, under Castanea sativa, 26 Sept. 2020, A. Vizzini (TO AV260920). AMANITA. Subgenus Amanita - Amanita argentea. Italy: EmiliaRomagna, Ronchi (Monterenzio, Bologna), mixed forest with Quercus pubescens and Q. cerris, 04 July 1994, G. Consiglio (AMB 18720). Subgenus Lepidella - Amanita phalloides. Italy: Trentino, Costasavina (Pergine Valsugana, Trento), mixed deciduous and coniferous forest, 28 Sept. 2005, G. Consiglio (AMB 18719).

\section{Discussion}

\section{Spore amyloidity}

Species identification of the Tricholoma collections analyzed, based on pileus colour, structure of the pileipellis, presence/absence of clamp-connections, size and shape of the basidiospores, was supported by molecular data (Fig. 2). All of the eighteen collections studied (seventeen species), which include taxa representative of all the ten sections recognized in Tricholoma in Europe by Christensen and Heilmann-Clausen (2013) and Heilmann-Clausen et al. (2017), showed spores with a positive reaction in Melzer's reagent under light microscope (Figs. 3, 4c-i, $5 c-f$ and $6 c-f$ ) by using the preheating and the CM30 and CM120 methods (Table 1). The spore print of $T$. josserandii shows an amyloid reaction when treated with a drop of Melzer's (Fig. 8).

The type of soaking medium used does not appear to have any influence on the iodine reactions of the spore wall, but the use of $\mathrm{KOH}$ is not recommended because it can sometimes induce distortions in the shape of some spores; furthermore, if the excess $\mathrm{KOH}$ is not well removed, hydroxide ions can react with iodine producing cloudy troublesome precipitates. Instead, very important factors are the preheating or not of the sample and the immersion time of the spores in Melzer's solution.

With the new method (HM), the amyloid reaction is also evident at the apex of the basidia and sterigmata (Fig. 3a-d). This would seem to indicate structural continuity and an identity of chemical composition between the basidial apex wall and the spore wall. By contrast, the amyloidity test of the same above-mentioned seventeen species, using the classical method (without heating) with spores observed immediately after being mounted in Melzer's (CMi), produced no clear results (Table 1) (Figs. 4a-b, 5a-b and 6a-b).

However, this is hardly surprising because in the monographic works and in the most important papers dealing with Tricholoma worldwide, spores are always reported as inamyloid (Kühner and Maire 1934; Hesler 1958; Horak 1964; Stevenson 1964; Singer (1966); Huijsman 1968; Gulden 1969, 1992; Ovrebo and Tylutki 1975; Bigelow 1979; Ovrebo and Smith 1979; Kost 1981, 1984; Clémençon 1983; Bon 1984, 1991; Ovrebo 1986, 1989; Hongo 1988; Kytövuori 1988; Riva 1988, 1998, 2003; Shanks 1998; Christensen and Noordeloos 1999; Noordeloos and Christensen 1999; Kalamees 2001; Deng and Yao 2005; Galli 2005; Yu et al. 2006; Christensen and Heilmann-Clausen 2008, 2012, 2013; Jargeat et al. 2010; Kirby 2012; Bessette et al. 2013; Hosen et al. 2016; Heilmann-Clausen et al. 2017; Trudell et al. (2017); Yang et al. 2017; Ovrebo and Hughes 2018; Reschke et al. 2018; Ovrebo et al. 2019; Şen and Alli 2019).

As indicated by Clémençon (2000, 2012), pitfalls in assessment of spore amyloidity may be due to several causes, among which first and foremost allowing insufficient time for the reaction to complete. In Mycena, he observed that, leaving the sample in Melzer's for at least $20 \mathrm{~min}$, the spores became black even in species which show very weak reactions (barely discernible under the microscope) when examined in Melzer's after a short time. Consequently, we tested the eighteen collections also following Clémençon's advice. First, the samples were soaked for 2 min in $5 \%$ ammonia (Fig. $4 \mathrm{c}-\mathrm{g}$ ) or for $2 \mathrm{~h}$ in tap water (Fig. $4 \mathrm{~h}-\mathrm{i}$ ), then they were transferred to, and squashed in Melzer's. No amyloid spores could be observed immediately after squashing, but $30 \mathrm{~min}$ later some amyloid spores were clearly visible (Fig. 4c-g). Re-observed after an additional $30 \mathrm{~min}$, the number of amyloid spores and 

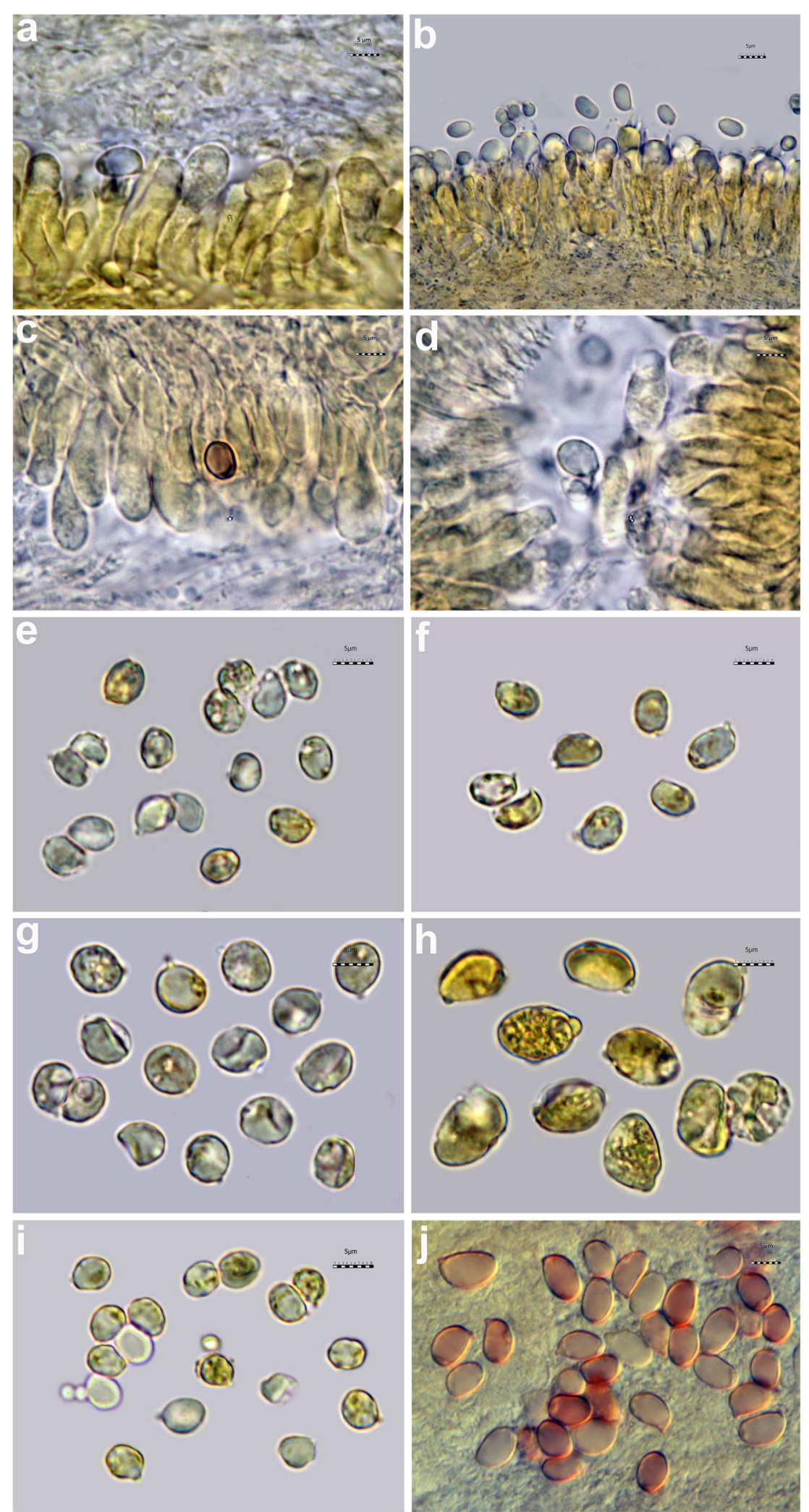

Fig. 3 Spores and basidia of Tricholoma species observed in Melzer's after heating. a-b T. argyraceum (AMB 17211) c-d T. josserandii (AMB 17407) e $T$. caligatum (AMB 17231) $\mathbf{f} T$. frondosae (AMB 17243) $\mathbf{g} T$. fucatum (AMB 17300) $\mathbf{h}$ T. inamoenum (AMB 17367) $\mathbf{i} T$. orirubens (AMB 17410) $\mathbf{j} T$. terreum (AMB 17444). Photos by L. Setti 


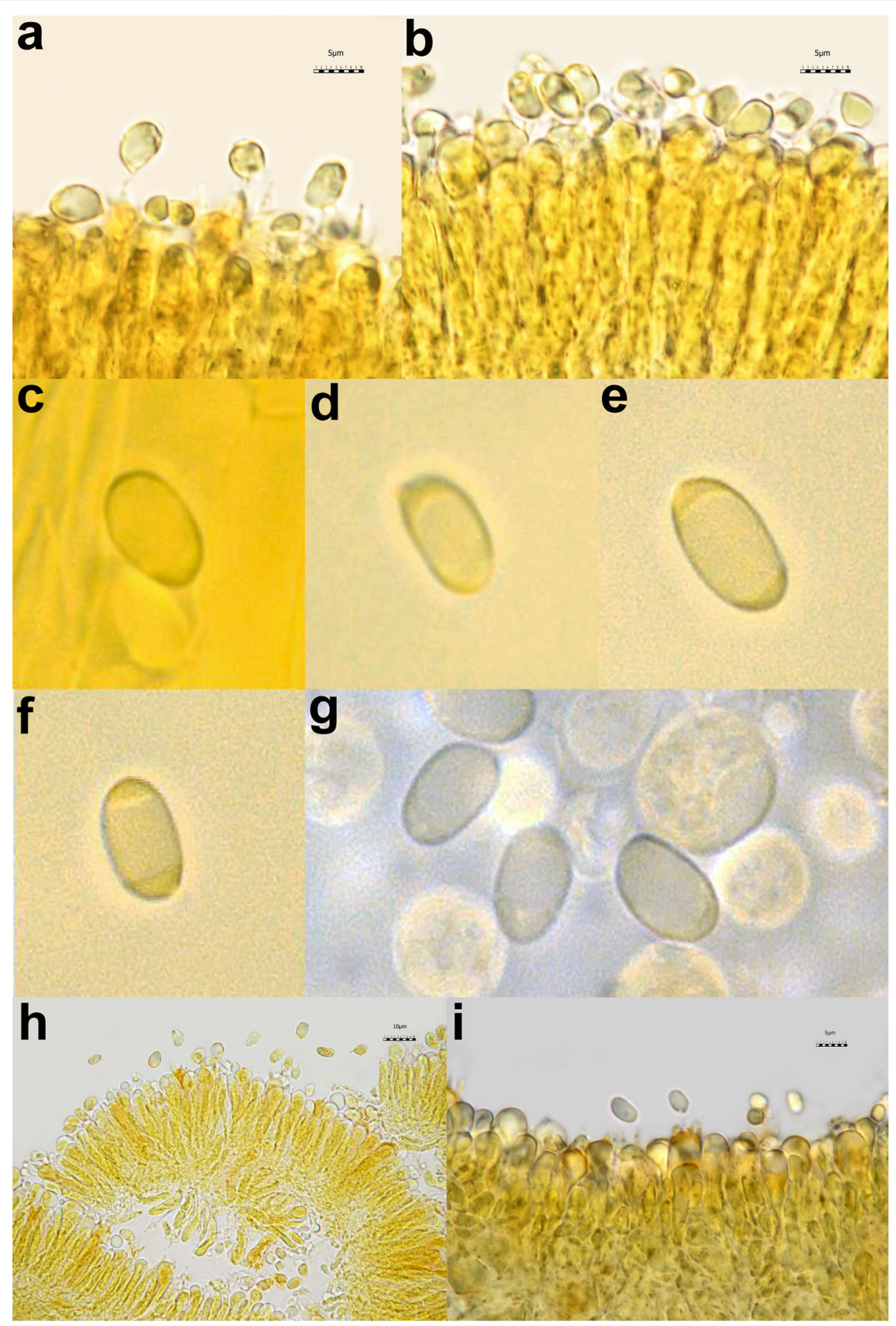

Fig. 4 Spores and basidia of Tricholoma argyraceum (AMB 17211) observed in Melzer's without pre-heating. a-b Sample soaked in 5\% ammonia and observed in Melzer's immediately after squashing. c-g Sample soaked in 5\% ammonia and observed after leaving the sample in Melzer's for 30 min. $\mathbf{h}-\mathbf{i}$ Sample soaked in tap water and observed leaving the sample in Melzer's for 30 min. Photos $\mathbf{a}-\mathbf{b}$ and $\mathbf{h}-\mathbf{i}$ by $L$. Setti; $\mathbf{c}-\mathbf{g}$ by H. Clémençon

the intensity of the amyloid reaction did not change and the same happened after $120 \mathrm{~min}$. Even with the CM30 method some basidia are apically amyloid (Fig. 4g-i).

Interestingly, the spores of $T$. josserandii and $T$. terreum demonstrated a dextrinoid reaction in Melzer's (Figs. $5 \mathrm{c}-\mathrm{f}$ and $6 \mathrm{c}-\mathrm{f}$ ), sometimes with small amyloid portions (Fig.
$3 \mathrm{c}-\mathrm{d}, \mathrm{j})$. To our knowledge this situation is absolutely unprecedented in the Agaricales and in all Basidiomycota.

It is not easy to explain the discrepancy between our results and those of all the other authors who have studied the genus Tricholoma. It might be possible that preheating the sample in Melzer's solution somehow allows 

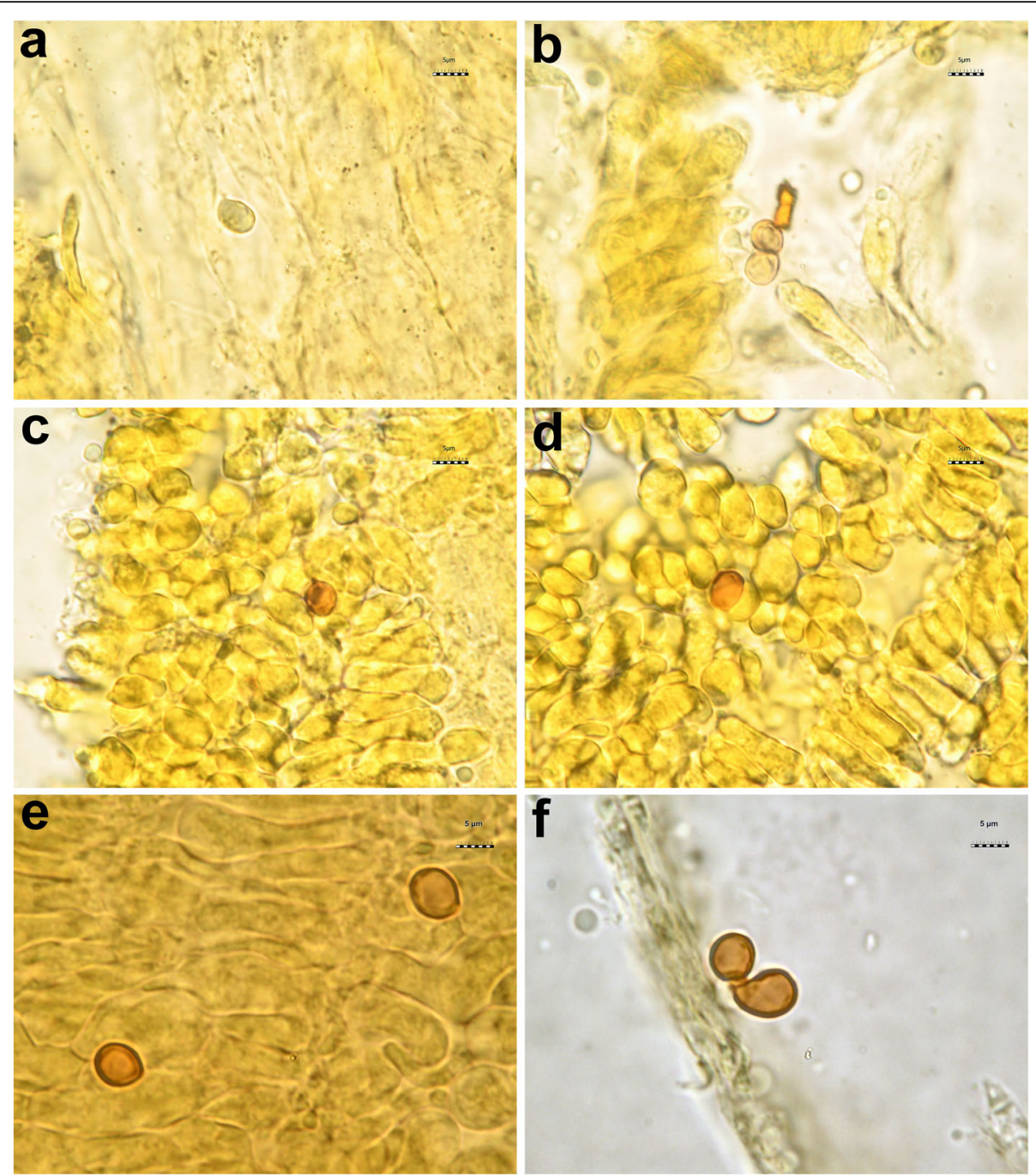

Fig. 5 Spores of Tricholoma josserandii (AMB 17407). a-d Observed in Melzer's without pre-heating. a-b Sample soaked in 5\% ammonia and observed in Melzer's immediately after squashing; c-d Sample soaked in 5\% ammonia and observed after leaving the sample in Melzer's for 30 min. e-f Observed in Melzer's with pre-heating. Photos by L. Setti

the starch-like wall material to better interact with the chemical agent and so make the colour reaction much more visible than when it is obtained in Melzer's at room temperature. The same may happen in unheated samples if the observation is made after waiting the right amount of time (at least $30 \mathrm{~min}$ ), that is giving iodine the necessary time to get in touch with the amyloid material in the sporal wall.

Whenever it is necessary to highlight the amyloidity of spores of species long known as clearly amyloid (see for example A. phalloides, Fig. 7a-e), any method proves effective. But whenever it is intended to analyze the spores of species with known dubious or weak amyloidity (e.g., Tricholoma in the present study; Musumecia, Pseudolaccaria, Alvarado et al. 2018; Vizzini et al. 2020) or of species not yet described or not tested, a negative reaction with the quick classical method (without pre-heating and observation of spores in Melzer's immediately after squashing, CMi) must be regarded as non-definitive and a stepwise process should be carried out.

In the description of tricholomatoid fungi and in preparing dichotomous keys we would like to suggest to indicate the spores which are amyloid after the CMi method as "immediately amyloid"; after CM30 as "tardily amyloid"; and after HM as "amyloid after pre-heating".

In conclusion, the authors recommend that the heating method described here, which is as effective as the CM30 but much faster, becomes of common use, and hope that in the future, taxonomic workers will routinely turn to "hot Melzer's" as a check when determining whether a species which appears to be iodine-negative is truly iodine-negative.

\section{Phylogenetic implications}

Tricholoma was first established as a tribus of the broad genus Agaricus (Fries 1821). Since then, it has been 

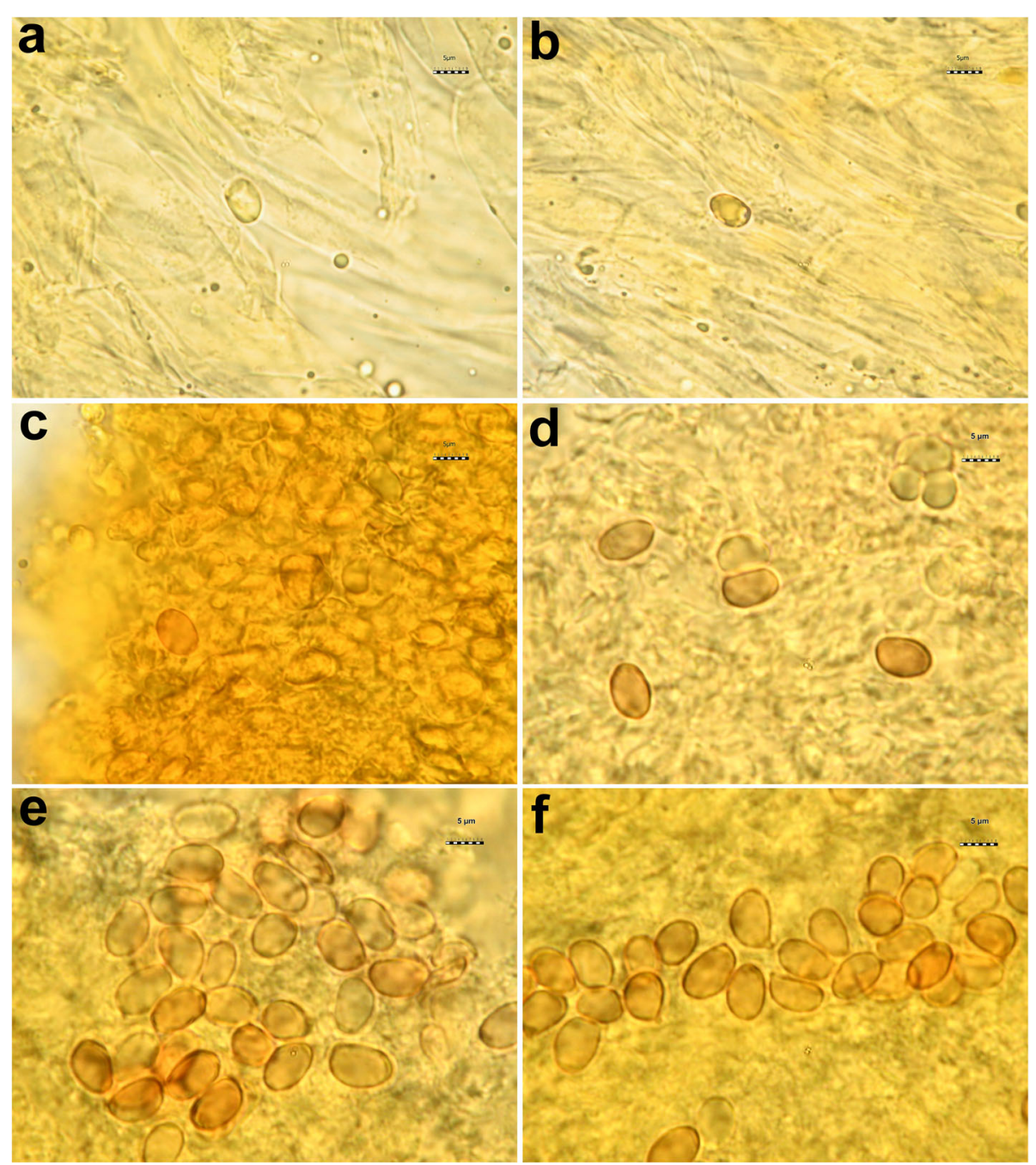

Fig. 6 Spores of Tricholoma terreum (AMB 17444). a-d Observed in Melzer's without pre-heating. a-b Sample soaked in 5\% ammonia and observed in Melzer's immediately after squashing; c-d Sample soaked in 5\% ammonia and observed after leaving the sample in Melzer's for 30 min. e-f Observed in Melzer's with pre-heating. Photos by L. Setti

restricted to ectomycorrhizal fungi with centrally stipitate, fleshy basidiomes with sinuate lamellae, and smooth, hyaline, inamyloid spores (Bon 1984, 1991; Singer 1986). Tricholoma is the type genus of the conserved family Tricholomataceae R. Heim ex Pouzar (McNeill et al. 2006). Tricholoma, the largest genus within the family (about 200 species worldwide according to Kirk et al. 2008), diverged from its saprotrophic sister clades Dermoloma and Pseudotricholoma during the late Eocene, 62.19 (36.26-92.0) Mya (Sánchez-García and Matheny 2017), and was first demonstrated to be monophyletic by Sánchez-García et al. (2014) based on a multigene analysis. They recovered a major clade, named Tricholomataceae s. str., which encompasses seven monophyletic clades corresponding to Leucopaxillus, Tricholoma, Pseudotricholoma, Porpoloma s. str., Dennisiomyces, Corneriella, and Albomagister. Sánchez-García et al. (2014) stated that Tricholoma and Albomagister are the only genera of the family Tricholomataceae s. str. with inamyloid spores. A similar phylogeny was included in Sánchez-García and Matheny (2017) and Corriol and Jargeat (2019) who recently reported the first collection of a species of Dennisiomyces in Europe. Also Pseudobaeospora and Dermoloma magicum together with four unidentified Dermoloma species were later placed in Tricholomataceae s. str. (Desjardin et al. 2014; SánchezGarcía and Matheny 2017). Pseudobaeospora is considered a genus with clearly dextrinoid spores (Singer 1963, 1986; Bas 2002, 2003; Voto 2009; Arauzo 2011) and D. magicum show amyloid spores (Arnolds 2002).

In the light of the data reported in the present paper, and considering that in the monospecific genus Albomagister has recently included a second species with faintly amyloid spores by Moreau et al. (2015), it seems 

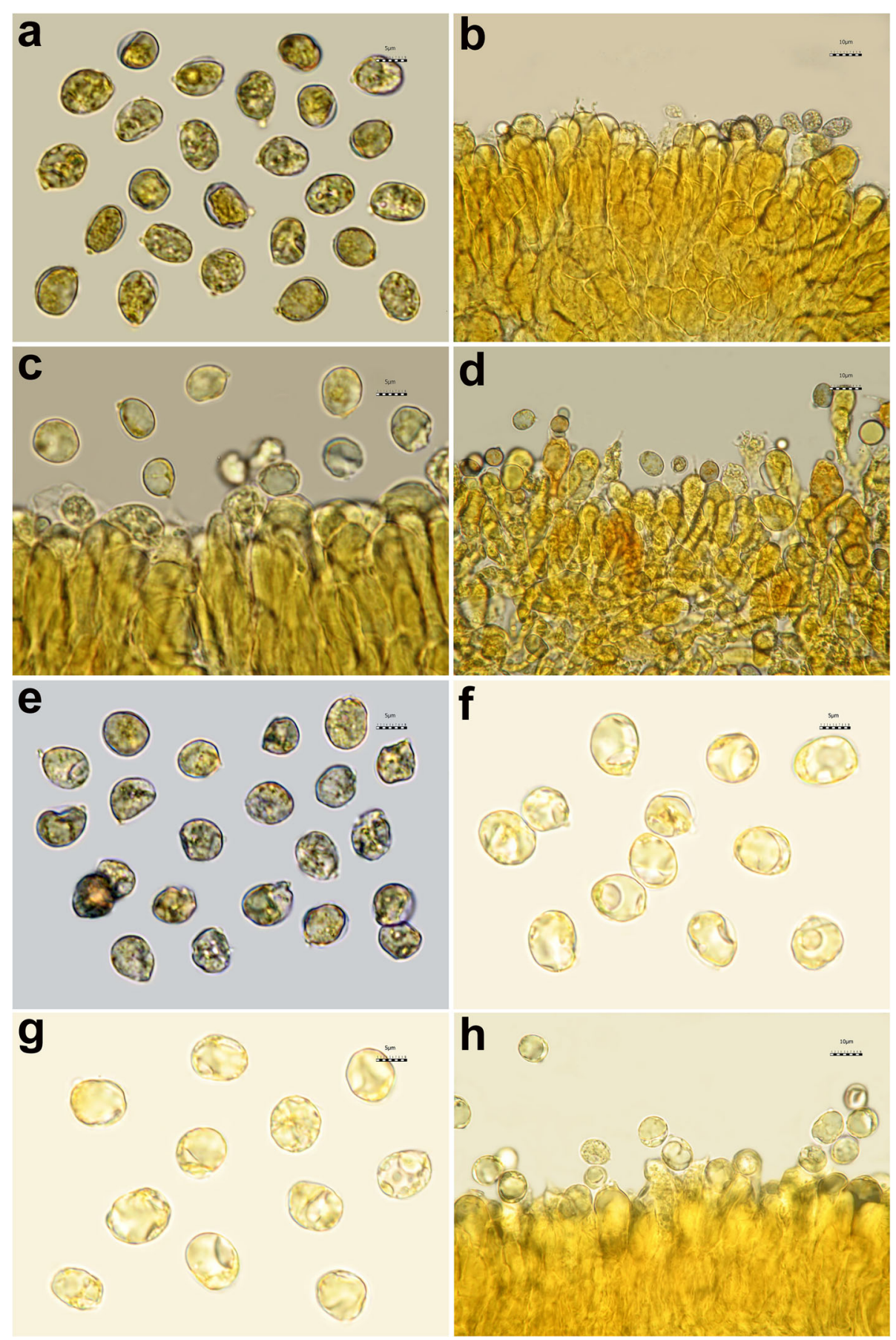

Fig. 7 Spores and basidia of Amanita phalloides (a-e, AMB 18719) and A. argentea (f-h, AMB 18720). a-c, $\mathbf{f}$ Observed in Melzer's without preheating, immediately after squashing (a soaked in tap water, $\mathbf{b}$, $\mathbf{f}$ in 5\% ammonia and $\mathbf{c}$ in 5\% potassium hydroxide). $\mathbf{d}-\mathbf{g}$ Observed in Melzer's without pre-heating after leaving the sample in Melzer's for 30 min (soaked in 5\% ammonia). e-h Observed in Melzer's with pre-heating. Photos by L. Setti

that a positive reaction of the spores in Melzer's reagent could be a character shared by all genera in Tricholomataceae s. str. and consequently be considered, in this case, a systematic marker at the family level.

\section{Conclusions}

At variance with what has been hitherto believed, the spores of the Tricholoma collections sampled here have proved to be amyloid when treated with a Melzer's 

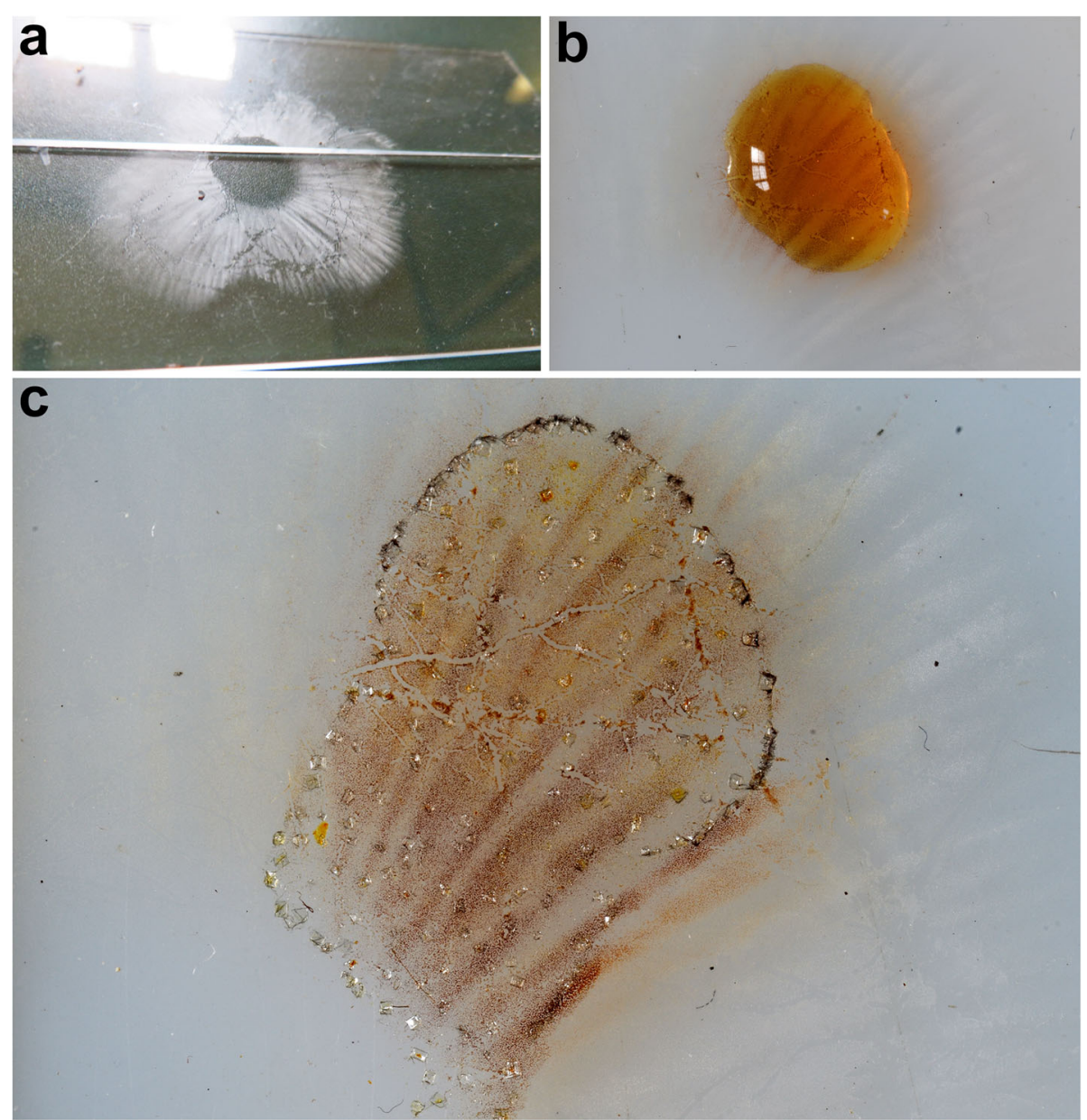

Fig. 8 Tricholoma josserandii (TO AV260920). a. Spore print on microscope slides. b. Drop of Melzer's placed on part of the spore print. c Part of the spore print observed after complete absorption/evaporation of the reagent $(2 \mathrm{~h}$ ). Photos by L. Setti

solution (original formulation) both with the classical method (without pre-heating), which involves an immersion in Melzer's for at least $30 \mathrm{~min}$, and a new method proposed in the present work, in which the sample is pre-heated. The soaking media appear to have no influence in inducing the reaction with the Melzer's solution.

Before being declared inamyloid, the spores of whatever species should be tested by all the methods suggested in this paper. It is hoped that the new method with preheating will be generally adopted by mycologists and that it will become widely used. A positive reaction of the spores with Melzer's reagent appears to be a character present in all genera of Tricholomataceae s. str.

\section{Abbreviations}

5.8S: 5.8 S ribosomal RNA; AMB: National Mycological Herbarium of the "Associazione Micologica Bresadola", Trento, Italy; Bl: Bayesian inference; BP: Bootstrap proportions; CM: Classical method for testing spore amyloidity without pre-heating; CMi: Classical method where spores are observed immediately after squashing; CM30: Classical method where spores are observed after 30 min. in Melzer's solution; CM120: Classical method where spores are observed after 120 min. in Melzer's solution;
CTAB: Cetyltrimethylammonium bromide; DNA: Deoxyribonucleic acid; HM: Heating method, a new method for testing spore amyloidity with preheating; ITS1: Internal transcribed spacer 1; ITS2: Internal transcribed spacer 2; Melzer's: Melzer's solution/reagent; ML: Maximum likelihood; Mya: Million years ago; nrlTS: Nuclear ribosomal internal transcribed spacer; PCR: Polymerase chain reaction; PP: Posterior probability

\section{Acknowledgments}

The authors are grateful to C. Lange (Natural History Museum of Denmark, Copenhagen) for sending the Tricholoma lascivum collection C-F-96230, to E. Grilli (Pescara) for improving the English text and to A. De Angelis (Urbino, Pesaro-Urbino), E. Franceschini (Bologna), M. Maletti (Pesaro, Pesaro-Urbino), G. Marasca (Pergine Valsugana, Trento), G. Perdisa (Casalecchio di Reno, Bologna), L. Polidori (Fano, Pesaro-Urbino), G. Simonini (Reggio Emilia), G. Spisni (Castenaso, Bologna), R. Trimarco (Casalecchio di Reno, Bologna) for helping in Tricholoma collections. H. Clémençon (Musée botanique cantonal, Lausanne) is also warmly thanked for providing helpful and stimulating suggestions and criticism. Sincere thanks to the anonymous reviewers of the manuscript.

\section{Adherence to national and international regulations} Not applicable.

\section{Authors' contributions}

AV, GC and LS conceived the study; all authors carried out sample collection; AV and GC performed molecular methods; GC performed phylogenetic 
analyses; AV and GC drafted the manuscript; all authors approved the final version of the manuscript.

\section{Funding}

Not applicable.

\section{Availability of data and materials}

Details of the availability of the data and material used in this study can be found within the text. DNA sequences were submitted to NCBI Genbank database (see Table 2). Dried specimens are deposited in the fungarium listed in the Methods section.

\section{Ethics approval and consent to participate}

Not applicable.

\section{Consent for publication}

Not applicable.

\section{Competing interests}

The authors declare no competing interests.

\section{Author details}

${ }^{1}$ Department of Life Sciences and Systems Biology, University of Torino and Institute for Sustainable Plant Protection (IPSP-SS Turin), C.N.R, Viale P.A. Mattioli, 25, I-10125 Torino, Italy. ${ }^{2}$ Bologna, Italy. ${ }^{3}$ Mantova, Italy.

Received: 19 May 2020 Accepted: 15 October 2020

\section{Published online: 11 November 2020}

\section{References}

Agerer R (1990) Studies on Ectomycorrhizae XXIV. Ectomycorrhizae of Chroogomphus helveticus and C. rutilus (Gomphidiaceae, Basidiomycetes) and their relationship to those of Suillus and Rhizopogon. Nova Hedwigia 50(1-2): $1-63$

Agerer R (1999) Never change a functionally successful principle: the evolution of Boletales s.l. (Hymenomycetes, Basidiomycota) as seen from below-ground features. Sendtnera 6:5-91

Agerer R (2018) Subphylum Agaricomycotina Doweld. In: Frey W (ed) Syllabus of plant families - a. Engler's syllabus der Pflanzenfamilien, part 1/3 Basidiomycota and Entorrhizomycota. Schweizerbart Science Publishers, Stuttgart, pp 130-444

Ainsworth GC (1961) Ainsworth and Bisby's dictionary of the Fungi. CMI, Kew

Altschul SF, Gish W, Miller W, Myers EW, Lipman DJ (1990) Basic local alignment search tool. Journal of Molecular Biology 215:403-410

Alvarado P, Moreau P-A, Dima B, Vizzini A, Consiglio G, Moreno G, Setti L, Kekki T, Huhtinen S, Liimatainen K, Niskanen T (2018) Pseudoclitocybaceae fam. Nov (Agaricales, Tricholomatineae), a new arrangement at family, genus and species level. Fungal Diversity 90(1):109-133

Ammirati JF, Traquair JA, Horgen PA (1985) Poisonous mushrooms of the northern United States and Canada. University of Minnesota Press, Minneapolis

Arauzo S (2011) Estudios en el género Pseudobaeospora. Errotari 8:14-37

Arnolds E (2002) Dermoloma magicum spec. nov., a grassland fungus mimicking Porpoloma metapodium. Persoonia 17(4):665-668

Bailey JM, Whelan WJ (1961) Physical properties of starch. I. Relationship between iodine stain and chain length. The Journal of Biological Chemistry 236:969973

Baral HO (1987a) Lugol's solution/IKI versus Melzer's reagent: hemiamyloidity, a universal feature of the ascus wall. Mycotaxon 29:399-450

Baral HO (1987b) Der Apikalapparat der Helotiales. Eine lichtmikroskopische Studie über Arten mit Amyloidring. Zeitschrift für Mykologie 53(1):119-136

Baral HO (2007) Über die Jodreaktion bei Ascomyceten. Tintling 12(2):16-22

Baral HO (2009) lodine reaction in Ascomycetes: why is Lugol's solution superior to Melzer's reagent? http://www.gbif-mycology.de/HostedSites/Baral/ lodineReaction.htm (Accessed 26 July 2020)

Bas C (2002) A reconnaissance of the genus Pseudobaeospora in Europe I. Persoonia 18(1):115-122

Bas C (2003) A reconnaissance of the genus Pseudobaeospora in Europe $\|$. Persoonia 18(2):163-199
Bas C, Kuyper TW, Noordeloos ME, Vellinga EC (eds) (1988) Flora Agaricina Neerlandica, volume 1: critical monographs on families of Agarics and Boleti occurring in the Netherlands. A.A. Balkema Publishers, Brookfield Basso MT (2005) Manuale di microscopia dei funghi, vol 1. Mykoflora, Alassio Bertoft E (2017) Understanding starch structure: recent progress. Agronomy 7:56 Bessette AE, Bessette AR, Roody WC, Trudell SA (2013) Tricholomas of North America: a mushroom field guide. University of Texas Press, Austin Bigelow HE (1979) A contribution to Tricholoma. Beihefte zur Sydowia 8:54-62 Bigelow HE (1982) North American species of Clitocybe. I. Beihefte zur Nova Hedwigia 72:1-280

Blackwell M, David C, Barker SA (2001) The presence of glycine betaine and the dextrinoid reaction in basidiomata. Harvard Papers in Botany 6(1):35-41

Blackwell M, Kinney AJ, Radford PT, Dugas CM, Gilbertson RL (1985) The chemical basis of Melzer's reaction. Mycological Society of America Newsletter 36(1):18

Bluhm TL, Zugenmaier P (1981) Detailed structure of the VH-amylose-iodine complex: a linear polyiodide chain. Carbohydrate Research 89:1-10

Bon M (1984) Les Tricholomes de France et d'Europe occidentale. Encyclopeéie Mycologique 36. Lechevalier, Paris

Bon M (1991) Tricholomataceae (Fayod) Heim (1ère partie) (Tricholomoideae et Leucopaxilloideae). Flore Mycologique d'Europe Vol. 2. Les Tricholomes et Ressemblants. Documents Mycologiques Mémoire Hors Série. Amiens, France

Boudier É (1885) Nouvelle classification naturelle des Discomycètes charnus. Bulletin Société Mycologique de France 1:91-120

Boudier É (1905-1910) Icones Mycologicae. Libraire des Sciences Naturelles, Paris

Boursier J (1925) Leucopaxillus nov. gen. Bulletin Société Mycologique de France 41:391-393

Brunori A, Buischio A, Cassinis A (1985) Introduzione allo studio dei funghi. Editrice II Libro, Roma

Calissendorff J, Falhammar H (2017) Lugol's solution and other iodide preparations: perspectives and research directions in Graves' disease. Endocrine 58(3):467-473

Charbonnel J (2004) Les réactifs mycologiques, Tome II: Les réactifs microchimiques. Ed. J.-C. David-Rogeat, Bienne, Switzerland

Chen J-F (1990) Effects of amylose and amylopectin on the functional properties of starch. Retrospective Theses and Dissertations. 16727. https://lib.dr.iastate. edu/rtd/16727

Christensen M, Noordeloos ME (1999) Notulae ad floram agaricinam neerlandicam XXXVI-Tricholoma. Persoonia 17:295-317.

Christensen M, Heilmann-Clausen J (2008) Tricholoma (Fr.) P. Kumm. In: Knudsen H, Vesterholt J (eds) Funga Nordica. Nordsvamp, Copenhagen, pp 413-429

Christensen M, Heilmann-Clausen J (2012) Tricholoma (Fr.) P. Kumm. In: Vesterholt J, Knudsen H (eds) Funga Nordica, 2nd edn. Nordsvamp, Copenhagen, pp 494-510

Christensen M, Heilmann-Clausen J (2013) The genus Tricholoma. Fungi of Northern Europe, vol 4. Svampetryk, Denmark

Clémençon H (1983) Die Erdritterlinge und ihre nächst verwandten Arten aus der Gattung Tricholoma, Sektion Tricholoma. Mycologia Helvetica 1:17-30

Clémençon H (2000) Unfug mit Melzer. - Idiotie avec le Melzer. Schweizerische Zeitschrift für Pilzkunde 78(6):276-278

Clémençon H (2012) Amyloide und dextrinoide Sporenwände noch einmal. Schweizerische Zeitschrift für Pilzkunde 90(2):53-55

Common RS (1991) The distribution and taxonomic significance of lichenan and isolichenan in the Parmeliaceae (lichenized Ascomycotina), as determined by iodine reactions. I. Introduction and methods. II. The genus Alectoria and associated taxa. Mycotaxon 41(1):67-112

Corriol G, Jargeat P (2019) Morphological and phylogenetical description of Dennisiomyces fibrillosus sp. nov. (Agaricales, Tricholomataceae) from the Pyrenees and the first record of the genus in Europe. Phytotaxa 405(5): 226-236

Crawshay R (1930) Spore ornamentation of the Russulas. Bailliere, Tindalland Cox Currey $F$ (1859) On the existence of amorphous starch in a new tuberaceous fungus. Proceedings of the Royal Society of London 9:119-123

Deng H, Yao YJ (2005) Revision of three species of Tricholoma reported from China. Mycotaxon 92:77-84

Dentinger BTM, Gaya E, O'Brien H, Suz LM, Lachlan R, Díaz-Valderrama JR, Koch RA, Aime MC (2016) Tales from the crypt: genome mining from fungarium specimens improves resolution of the mushroom tree of life. Biological Journal of the Linnean Society 117:11-32

Desjardin DE, Hemmes DE, Perry BA (2014) A ruby-colored Pseudobaeospora species is described as new from material collected on the island of Hawaii. Mycologia 106(3):456-463

Dodd JL (1972) The genus Clavicorona. Mycologia 64(4):737-773 
Dodd JL, McCracken DA (1972) Starch in fungi. Its molecular structure in three genera and an hypothesis concerning its physiological role. Mycologia 64: $1341-1343$

Donk MA (1964) A conspectus of the families of Aphyllophorales. Persoonia 3: 199-324

Du X, An H, Liu Z, Yang H, Wei L (2014) Probing starch-iodine interaction by atomic force microscopy. Scanning 36(4):394-400

Edgar RC (2004) MUSCLE: multiple sequence alignment with high accuracy and high throughput. Nucleic Acids Research 32(5):1792-1797

Erb B, Matheis W (1983) Pilzmikroskopie. Präparation und Untersuchung von Pilzen. Franckh'sche Verlagshandlung, Stuttgart

Frey W (2016) Syllabus of plant families - A. Engler's syllabus der Pflanzenfamilien Part 1/2: Ascomycota. Gebrüder Borntraeger Verlag, Stuttgart

Fries E (1821) Systema mycologicum. Vol. 1. Ex officina Berlingiana, Lundae.

Galli R (2005) I tricolomi: atlante pratico-monografico per la determinazione del genere Tricholoma (Fr.) Staude. Edizioni Edinatura, Milano

Gardes M, Bruns TD (1993) ITS primers with enhanced specificity for Basidiomycetes - application to the identification of mycorrhizae and rusts. Molecular Ecology 2:113-118

Gilbert EJ (1940) Amanitaceae. Bresadola Iconographia Mycologica 27(1):1-200

Gilbert EJ, Kühner R (1928) Recherches sur les spores des amanites. Bulletin de la Société Mycologique de France 44(2):149-154

Ginns J (1998) Genera of the North American Corticiaceae sensu lato. Mycologia 90:1-35

Gulden G (1969) Musseronflora. Universitetsforlaget, Norway. Musseronflora: slekten Tricholoma (Fr. ex Fr.) Kummer sensu lato. Universitetsforlaget, Oslo, Norway

Gulden G (1992) Tricholoma (Fr.) Staude. In: Hansen L, Knudsen H (eds) Nordic Macromycetes, vol 2. Nordsvamp, Copenhagen, pp 183-191

Halbwachs H, Bässler C (2015) Gone with the wind - a review on basidiospores of lamellate agarics. Mycosphere 6(1):78-112

Harder CB, Lodge DJ, Petersen RH, Hughes KW, Blanco JC, Frøslev TG, Læssøe T (2012) Amyloidity is not diagnostic for species in the Mycena pearsoniana complex (Mycena sectio Calodontes). Mycological Progress 11:725-732

Harmaja H (2002) Amylolepiota, Clavicybe and Cystodermella, new genera of the Agaricales. Karstenia 42(2):39-48

Heilmann-Clausen J, Christensen M, Frøslev TG, Kjøller (2017) Taxonomy of Tricholoma in northern Europe based on ITS sequence data and morphological characters. Persoonia 38:38-57

Hesler LR (1958) Southeastern Agaricales II. Studies of Tricholoma types. Journal of the Tennessee Academy of Science 33:186-191

Hjortstam K, Larsson K-H, Ryvarden L (1988) The Corticiaceae of North Europe. With drawings by John Eriksson volume 1. Introduction and keys. Fungiflora, Oslo

Hongo T (1988) On the genus Tricholoma of Japan. Transactions of the Mycological Society of Japan 29:441-447

Horak E (1964) Fungi Austroamericani I - Tricholoma. Sydowia 17:153-167

Horak E (2005) Röhrlinge und Blätterpilze in Europa. Elsevier GmbH, Spektrum Akademischer Verlag, Heidelberg

Hosen MI, Li T-H, Li T, Zhong X-J, Chen Y (2016) Tricholoma sinoacerbum, a bitter species from Guangdong Province of China. Mycoscience 57:233-238

Huijsman HSC (1968) Observations sur les Tricholomataceae 1. Schweizerische Zeitschrift für Pilzkunde 46:143-153

Imler L (1950) Recherches sur les bolets. Bulletin de la Société Mycologique de France 66(4):177-203

Immel S, Lichtenthaler FW (2000) Molecular modeling of saccharides, part XXIV. The hydrophobic topographies of amylose and its blue iodine complex. Starch/Stärke 52:1-8

Jargeat P, Martos F, Carriconde F, Gryta H, Moreau P-A, Gardes M (2010) Phylogenetic species delimitation in ectomycorrhizal fungi and implications for barcoding: the case of the Tricholoma scalpturatum complex. Molecular Ecology 19:5216-5230

Jülich W (1984) Die Nichtblätterpilze, Gallertpilze und Bauchpilze. In: Gams H (ed) Kleine Kryptogamentflora, Part Ilb/1. Gustav Fischer Verlag, Stuttgart

Jülich W, Stalpers JA (1980) The resupinate non-poroid Aphyllophorales of the temperate northern hemisphere. Verhandelingen der Koninklijke Nederlandse Akademie van Wetenschappen, Afd. Natuurkunde, Tweede Reeks, Deel 74:1-335

Kalamees K (2001) Taxonomy and ecology of the species of the Tricholoma equestre group in the Nordic and Baltic countries. Folia Crypt Estonica 38:1323
Kirby G (2012) The genus Tricholoma in Britain. Private Print, UK

Kirk P, Cannon PF, Minter DW, Stalpers JA (2008) The dictionary of fungi. CAB International, Wallingford

Knudsen H, Vesterholt J (2018) Funga Nordica: Agaricoid, boletoid, clavarioid, cyphelloid and gastroid genera. Nordsvamp, Copenhagen

Kohn LM, Korf RP (1975) Variation in ascomycete iodine reactions: $\mathrm{KOH}$ pretreatment explored. Mycotaxon 3(1):165-172

Kost G (1981) Vergleichende morphologische, anatomische und feinstrukturelle Merkmalsstudien an Arten der Gattung Tricholoma (Fr.) Staude, Sektion Genuina (Fr.) Sacc. [PhD dissertation]. Eberhard Karls-Universität, Tübingen, Germany

Kost G (1984) Megatricholoma nov. gen. Eine neue agaricoide Gattung mit verwandtschaftlichen Beziehungen zu Arten anderer Organisationsstufen der Homobasidiomyzeten. Sydowia 37:53-74

Kühner R (1926a) Contribution à l'Étude des Hyménomycètes et spécialement des agaricacées. Le Botaniste 17:1-215

Kühner R (1926b) Remarques sur les genres Lepista et Leucopaxillus. Bulletin Mensuel de la Société Linnéenne de Lyon 5:125-126

Kühner R (1931) Utilisation de la réaction iodée dans la classification des Mycena. Bulletin Mensuel de la Société Linnéenne de Lyon 8:122-127

Kühner R (1936a) Sur la reaction a l'iode des parois des hyphes du carpophore des Mycena. Comptes Rendus. Académie des Sciences 203:1287-1290

Kühner R (1936b) Recherches sur le genre Lepiota. Bulletin de la Société Mycologique de France 52:175-238

Kühner R (1938) Le genre Mycena (Fries) Étude cytologique et systématique des espèces d'Europe et d'Amérique du Nord. Encyclopédie Mycologique 10:1710

Kühner R (1980) Les Hyménomycètes agaricoïdes (Agaricales, Tricholomatales, Pluteales, Russulales) - Étude générale et classification. Numéro special du Bulletin de la Société Linnéenne de Lyon, 49e Annèe, Lyon

Kühner R (1984) Some mainlines of classification in the gill fungi. Mycologia 76(6):1059-1074

Kühner R, Maire R (1934) Étude de la réaction de la membrane sporique à l'iode dans les divers genres d'Agarics leucospores. Bulletin de la Société Mycologique de France 50:9-24

Kühner R, Romagnesi H (1953) Flore analytique des champignons supérieurs. Masson, Paris

Kytövuori I (1988) The Tricholoma caligatum group in Europe and North Africa. Karstenia 28:65-77

Langeron M (1945) Précis de mycologie. Masson, Paris

Largent DL, Johnson D, Watling R (1977) How to identify mushrooms to genus III: microscopic features. Mad River Press, Arcata

Lavorato C, Vizzini A, Ge Z-W, Contu M (2015) Redescription of Clitocybe umbrinopurpurascens (Basidiomycota, Agaricales) and revision of Neohygrophorus and Pseudoomphalina. Phytotaxa 219(1):43-57

Lecomte M (2017) Microscopie \& champignons. AMFB

Leonard LM (2006) Melzer's, Lugol's or iodine for identification of white-spored Agaricales? Mcllvainea 16(1):43-51

Li GJ, Hyde HD, Zhao RL et al (2016) Fungal diversity notes 253-366: taxonomic and phylogenetic contributions to fungal taxa. Fungal Diversity 78:1-237

Locquin M, Langeron M (1978) Manuel de microscopie. Masson, Paris Ludwig E (2012) Pilzkompendium, band 3. Fungicon Verlag, Berlin

Malençon G (1931) Considerations sur les spores des Russules et des Lactaires. Bulletin de la Société Mycologique de France 47:72-85

Matheny PB, Curtis JM, Hofstetter V, Aime MC, Moncalvo JM, Ge ZW, Slot JC, Ammirati JF, Baroni TJ, Bougher NL, Hughes KW, Lodge DJ, Kerrigan RW, Seidl MT, Aanen DK, DeNitis M, Daniele GM, Desjardin DE, Kropp BR, Norvell LL, Parker A, Vellinga EC, Vilgalys R, Hibbett DS (2006) Major clades of Agaricales: a multilocus phylogenetic overview. Mycologia 98(6):982-995

McCracken DA, Dodd JL (1971) Molecular structure of starch-type polysaccharides from Hericium ramosum and Hericium coralloides. Science 174:419

McCracken DA, Nadakavukaren MJ, Dodd JL (1973) Starch in Fungi. II. Induction of amyloidity in members of diverse genera. American Journal of Botany 60(9):940-943

McNeill J, Barrie FR, Burdet HM, Demoulin V, Hawksworth DL, Marhold K, Nicolson DH, Prado J, Silva PC, Skog JE, Wiersema JH, Turland NJ (eds) (2006) International Code of Botanical Nomemclature (Vienna Code). Regnum Vegetabile 146. ARG Gantner, Ruggell.

Meixner A (1975) Chemische Farbreaktionen von Pilzen. Verlag J. Cramer, Vaduz 
Melzer MV (1924) L'ornementation des spores de Russules. Bulletin de la Société Mycologique de France 40:78-81

Metrod G (1932) Coloration par l'iode des spores des Lepiotes. Bulletin de la Société Mycologique de France 48:324

Meyer A (1883) Das Chlorophyllkorn in chemischer, morphologischer und biologischer Beziehung. A. Felix, Leipzig

Miller MA, Pfeiffer W, Schwartz T (2010) Creating the CIPRES science gateway for inference of large phylogenetic trees. In: Proceedings of the Gateway Computing Environments Workshop (GCE), 14 November 2010, New Orleans, pp. 1-8

Miller OK (1964) Monograph of Chroogomphus (Gomphidiaceae). Mycologia 56: 526-549

Moreau F (1930) L'ornamentation des spores des Russules. Bulletin de la Société Botanique de France 77:310-324

Moreau P-A, Bellanger J-M, Biancardini S, Richard F (2015) Albomagister alesandrii sp. nov., un nouvel element du patrimoine naturel de la Corse. Bulletin semestriel de la Fédération des Associations Mycologiques Méditerranéennes 48:7-14

Morton JB (1986) Effects of mountants and fixatives on wall structure and Melzer's reaction in spores of two Acaulospora species (Endogonaceae). Mycologia 78(5):787-794

Moser M (1961) Die Gattung Phlegmacium (Schleimköpfe). In: Die Pilze Mitteleuropas IV. Verlag Julius Klinkhardt. Bad Heilbrunn/Obb

Moser M (1967) Röhrlinge und Blätterpilze. 3. Aufl. Kleine Kryptogamenflora Mitteleuropas. Bd. 2b/2. G. Fischer. Stuttgart

Moser M (1978) Röhrlinge und Blätterpilze. 4. Aufl. Kleine Kryptogamenflora Mitteleuropas. Bd. 2b/2. G. Fischer, Stuttgart

Moulik SP, Gupta S (1984) Stability of the amylose-iodine complex. Carbohydrate Research 125(2):340-342

Mullis KB, Faloona FA (1987) Specific synthesis of DNA in vitro via a polymerasecatalyzed chain reaction. Methods in Enzymology 155:335-350

Murdoch KA (1992) The amylose-iodine complex. Carbohydrate Research 233 161-174

Murray MG, Thompson WF (1980) Rapid isolation of high molecular weight plant DNA. Nucleic Acids Research 8(19):4321-4325

Nannfeldt JA (1976) lodine reactions in ascus plugs and their taxonomic significance. Transactions of the British Mycological Society 67(2):283-287

Neville P, Poumarat S (2004) Amaniteae: Amanita, Limacella \& Torrendia. Edizioni Candusso, Alassio

Noordeloos ME, Christensen M (1999) Tricholoma. In: Bas C, Kuyper TW, Noordeloos ME, Vellinga EC (eds) Flora Agaricina Neerlandica, vol 4. Balkema, Rotterdam, pp 107-148

Nylander W (1865) Ad historiam reactionis iodi apud Lichenes et Fungos notula. Flora 4(30):465-468

Okuda M, Hiramatsu T, Yasuda M, Ishigaki M, Ozaki Y, Hayashi M, Tominaga K, Chatani E (2020) Theoretical modeling for electronic structure of Polyiodide species included in a-Cyclodextrin. The Journal Physical Chemistry B. https:// doi.org/10.1021/acs.jpcb.0c01749

Orton PD (1960) New checklist of British agarics and boleti part III. Notes on genera and species. Transactions of the British Mycological Society 43:159439

Ovrebo C, Smith AH (1979) Studies on Tricholoma vaccinum and Tricholoma imbricatum in North America. Michigan Botanist 18:99-106

Ovrebo C, Tylutki EE (1975) New species and a new combination of Tricholoma (Agaricales) from the Pacific Northwest. Mycologia 67:75-82

Ovrebo CL (1986) Three new species of Tricholoma with a description of Tricholoma luteomaculosum. Mycologia 78(3):418-425

Ovrebo CL (1989) Tricholoma, subgenus Tricholoma, section Albidogrisea: north American species found principally in the Great Lakes Region. Canadian Journal of Botany 67:3134-3152

Ovrebo CL, Hughes KW (2018) Tricholoma smithii, a new species in the Pardinicutis complex from New Mexico and Colorado. North American Fungi 13:1-9

Ovrebo CL, Hughes KW, Halling RE (2019) Three new species of Tricholoma from Costa Rica. Phytotaxa 392(1):33-44

Patouillard N (1887) Contributions à l'étude des champignons extra-européens. Bulletin de la Société Mycologique de France 3(2):119-131

Patouillard N (1888) Quelques points de la classification des Agaricinées. Journal de Botanique 2:12-16

Redhead SA (1977) The genus Neolecta (Neolectaceae fam. Nov. Lecanorales, Ascomycetes) in Canada. Canadian Journal of Botany 55(3):301-306
Reschke K, Popa F, Yang ZL, Kost G (2018) Diversity and taxonomy of Tricholoma species from Yunnan, China, and notes on species from Europe and North America. Mycologia 110(6):1081-1109

Riva A (1988) Fungi Europaei Vol. 3, Tricholoma (Fr.) Staude. Edizioni Candusso, Alassio

Riva A (1998) Fungi non delineati V: Tricholoma (Fr.) Staude. Mykoflora, Alassio

Riva A (2003) Tricholoma (Fr.) Staude (Suppl.). Edizioni Candusso, Alassio

Rolland L (1887) De la coloration en bleu développée par l'iode sur divers champignons et notamment sur un agaric. Bulletin de la Société Mycologique de France 3:134-137

Rossman AY (1980) The iodine reaction: Melzer's vs. IKI. MSA Newsletter 31:22

Ryvarden L, Melo I (2014) Poroid fungi of Europe. Synopsis Fungorum 31:1-455

Saar I, Põldmaa K, Kõljalg U (2009) The phylogeny and taxonomy of genus Cystoderma (Agaricales) based on evidence from nuclear ITS and LSU sequences. Mycological Progress 8(1):59-73

Sánchez-García M, Matheny PB (2017) Is the switch to an ectomycorrhizal state an evolutionary key innovation in mushroom-forming fungi? A case study in the Tricholomatineae (Agaricales). Evolution 71(1):51-65

Sánchez-García M, Matheny PB, Palfner G, Lodge DJ (2014) Deconstructing the Tricholomataceae (Agaricales) and introduction of the new genera Albomagister, Corneriella, Pogonoloma and Pseudotricholoma. Taxon 63:9931007

Scambler R, Niskanen T, Assyov B, Ainsworth AM, Bellanger JM, Loizides M, Moreau PA, Kirk PM, Liimatainen K (2018) Diversity of Chroogomphus (Gomphidiaceae, Boletales) in Europe, and typification of C. rutilus. IMA Fungus 9:271-290

Sen I, Alli H (2019) Tricholoma (Fr.) Staude in the Aegean region of Turkey. Turkish Journal of Botany 43:817-830

Shanks KM (1998) The Agaricales (gilled fungi) of California. 11. Tricholomataceae II. Tricholoma. Mad River Press, Eureka

Shen X, Bertoft E, Zhang G, Hamaker BR (2013) lodine binding to explore the conformational state of internal chains of amylopectin. Carbohydrate Polymers 98(1):778-783

Singer R (1932) Monographie der Gattung Russula. Beihefte zum Botanischen Centralblatt 49(2):205-380

Singer R (1938) Notes sur quelques Basidiomycetes. Revised Mycology 3:187-199

Singer R (1944) Notes on taxonomy and nomenclature of the polypores. Mycologia 36(1):65-69

Singer R (1951) The Agaricales in modern taxonomy. Lilloa 22:1-832

Singer R (1963) The delimitation of the genus Pseudobaeospora. Mycologia 55(1): $13-17$

Singer R (1965) Die Röhrlinge, Teil I. Boletaceae (ohne Boletoideae). Julius Klinkhardt verlag, Bad Heilbrunn

Singer R (1966) Monographs of south American Basidiomycetes, especially those of the east slope of the Andes and Brazil. IX. Tricholoma in Brazil and Argentina. Darwiniana 14:19-35

Singer R (1975) The Agaricales in modern taxonomy, 3rd edn. J. Cramer Verlag, Vaduz.

Singer R (1982) Flora Neotropica. Monograph 32. Hydropus (Basidiomycetes Tricholomataceae - Mycenae). The New York Botanical Garden, New York

Singer R (1986) The Agaricales in modern taxonomy, 4th edn. Koeltz Scientific Books, Königstein

Smith AH (1965) New and unusual Basidiomycetes with comments on hyphal and spore wall reactions with Melzer's solution. Mycopathologia et Mycologia Applicata 26(4):385-402

Smith AH, Zeller SM (1966) A preliminary account of the North American species of Rhizopogon. Memoirs of the New York Botanical Garden 14(2):1-178

Stalpers JA (1996) The aphyllophoraceous fungi II. Keys to the species of the Hericiales. Studies in Mycology 40:1-185

Stamatakis A (2014) RAxML version 8: a tool for phylogenetic analysis and postanalysis of large phylogenies. Bioinformatics 30:1312-1313

Stevenson G (1964) The Agaricales of New Zealand: V. Kew Bulletin 19:1-59

Takahashi K, Ono S (1972) Calorimetric study of amylose-iodine complex formation. Journal of Biochemistry 72(4):1041-1043

Tamura K, Stecher G, Peterson D, Filipski A, Kumar S (2013) MEGA6: molecular evolutionary genetics analysis version 6.0. Molecular Biology and Evolution 30:2725-2729

Trudell SA, Xu J, Saar I, Justo A, Cifuentes J (2017) North American matsutake: names clarified and a new species described. Mycologia 109:379-390

Tulasne LR, Tulasne C (1865) Selecta Fungorum Carpologia, vol 3. Imperatoris Jussu, in Imperiali Typographeo Excudebatur, Paris 
Tulloss RE (2020) Amanita argentea. In: Tulloss RE, Yang ZL (eds) Amanitaceae studies http://www.amanitaceae.org? Amanita+argentea ]. Accessed 14 Aug 2020

Tulloss RE, Possiel L (2020) Amanita phalloides. In: Tulloss RE, Yang ZL (eds) Amanitaceae studies [http://www.amanitaceae.org? Amanita+phalloides ]. Accessed 14 Aug 2020

Vizzini A, Consiglio G, Marchetti M, Alvarado P (2020) Insights into the Tricholomatineae (Agaricales, Agaricomycetes): a new arrangement of Biannulariaceae and Callistosporium, Callistosporiaceae fam. Nov., Xerophorus stat. Nov., and Pleurocollybia incorporated into Callistosporium. Fungal Diversity 101:211-259

Vizzini A, Simonini G, Ercole E, Voyron S (2014) Boletus mendax, a new species of Boletus sect. Luridi from Italy and insights on the B. luridus complex. Mycological Progress 13(1):95-109

Voto P (2009) Proposta di una sistematica infragenerica del Genere Pseudobaeospora, fondata su basi morfologiche, e note su due specie non ancora descritte. Rivista di Micologia 52(4):291-311

Watling R (1980) How to identify mushrooms to genus V. Mad River Press, Eureka

Webster J, Weber R (2007) Introduction to fungi, 3rd edn. Cambridge University Press, New York

White TJ, Bruns TD, Lee S, Taylor JW (1990) Amplification and direct sequencing of fungal ribosomal RNA genes for phylogenetics. In: Innis MA, Gelfand DH, Sninsky J, White TJ (eds) PCR protocols: a guide to methods and applications - a laboratory manual. Academic Press, San Diego, pp 315-322

Yang ZL, Ding X-X, Kost G, Rexer K-H (2017) New species in the Tricholoma pardinum complex from eastern Himalaya. Phytotaxa 305:1-10

Yu FQ, Wang Y, Liu PG (2006) Tricholoma lavendulophyllum, a new species from Yunnan, China. Mycotaxon 95:305-308

\section{Publisher's Note}

Springer Nature remains neutral with regard to jurisdictional claims in published maps and institutional affiliations.

Ready to submit your research? Choose BMC and benefit from:

- fast, convenient online submission

- thorough peer review by experienced researchers in your field

- rapid publication on acceptance

- support for research data, including large and complex data types

- gold Open Access which fosters wider collaboration and increased citations

- maximum visibility for your research: over $100 \mathrm{M}$ website views per year

At BMC, research is always in progress.

Learn more biomedcentral.com/submissions 